\title{
Grado de cobertura del muestreo de escarabajos coprófagos (Coleoptera: Scarabaeidae: Scarabaeinae) en Colombia
}

\author{
Jorge Ari Noriega ${ }^{1}$, Edgar Camero R. ${ }^{2}$, Jorge Arias-Buriticá ${ }^{3}$, Luis Carlos Pardo-Locarno ${ }^{4}$, José \\ Mauricio Montes ${ }^{5}$, Aldemar A. Acevedo ${ }^{6}$, Andrea Esparza ${ }^{7}$, Betselene Murcia Ordóñez ${ }^{8}$, Hector \\ Garcia $^{9}$ \& Cesil Solís ${ }^{10}$ \\ 1. Laboratorio de Zoología y Ecología Acuática - LAZOEA, Universidad de Los Andes, Bogotá, Colombia; \\ jnorieg@hotmail.com \\ 2. Departamento de Biología, Universidad Nacional de Colombia, Bogotá, Colombia; eecameror@unal.edu.co \\ 3. Universidad Nacional de Colombia, Bogotá, Colombia; joaariasbu@unal.edu.co \\ 4. Universidad del Pacífico, Buenaventura, Colombia; pardolc@gmail.com \\ 5. Universidad Nacional de Colombia, Medellín, Colombia; jmpamplonman@gmail.com \\ 6. Grupo de Ecología y Biogeografía, Facultad de Ciencias Básicas, Universidad de Pamplona, Pamplona, Colombia; \\ bioaldemar@gmail.com \\ 7. Universidad Industrial de Santander, Bucaramanga, Colombia; aesparzaleon@gmail.com \\ 8. Grupo BYDA, Universidad de la Amazonía, Florencia, Colombia; b.murcia@udla.edu.co \\ 9. Herbario UTMC, Universidad del Magdalena, Santa Marta, Colombia; coleopterocoprofago@yahoo.es \\ 10. Universidad del Atlántico, Fundación Hidrobiológica George Dahl, Barranquilla, Colombia; cesilsoli@yahoo.com
}

$$
\text { Recibido 05-II-2014. C Corregido 19-VII-2014. Aceptado 21-VIII-2014. }
$$

\begin{abstract}
Estimation of the sampling cover for dung beetles (Coleoptera: Scarabaeinae) in Colombia. The promotion of biodiversity conservation strategies must address the lack of information and the difficulty of identifying knowledge gaps that may facilitate our knowledge of different taxonomic groups. Dung beetles constitute one of those groups, despite having been proposed as an efficient bioindicator of environmental disturbance processes. In this work, we aimed to prepare a diagnosis on the state of knowledge of the subfamily Scarabaeinae, focusing on the cover sampling degree of this group in Colombia, with the purpose of identifying high-priority areas that will allow the completion of a national inventory. The work consisted of a bibliographical compilation using 12 referential databases and the examination of specimens deposited in 26 national collections. A total of 16940 individuals were examined, finding registers for 232 species from 386 localities. The respective distribution cover maps were presented, and the cover at a national level was $10.62 \%$. A historical analysis demonstrated a proliferation in the number of studies for the last three decades; nevertheless, a great proportion of unpublished works persists, resulting in only 64 sampled localities with published records. The localities with the greatest sampling efforts were RN La Planada, Lloro, AUN Los Estoraques, PNN Tinigua and Mariquita. Registries for all departments were available, and the best sampled ones were Cundinamarca, Antioquia, Valle del Cauca and Boyaca. The ecosystems with the greatest number of publications are the Andean pre mountain humid forest, followed by the Andean mountain humid forest and the Pacific humid forest. Other ecosystems with few studies included mangroves, desert zones, natural savannahs, palm swamps, paramos, flooding forests and agroforestry systems. The biogeographic region with the greatest number of localities was the Andean region, followed by Choco-Magdalenense and Amazonia. Our results showed that high levels of subsampling persist and that some zones lack registries, as in the case of some parks of the national system of protected areas. It is imperative that the sampling cover is extended at a national level, focusing all possible efforts on collecting in those subsampled regions that have high conservation importance, with the main goal of completing the listing of species and their distribution. Rev. Biol. Trop. 63 (1): 97-125. Epub 2015 March 01.
\end{abstract}

Key words: biogeographic regions, collections, geographic distribution, localities, sampling, Scarabaeidae. 
La ausencia o deficiencia de información autoecológica y sinecológica de las especies, gremios o comunidades es uno de las principales limitantes para el desarrollo de estrategias de conservación (Pimm et al., 1995, Margules \& Pressey, 2000). Son pocos los grupos taxonómicos que tienen un sistema completo de información que permita responder preguntas sobre la cantidad de estudios realizados, las regiones que necesitan más trabajo o sencillamente, que tengan identificados los vacíos de conocimiento que permitan avanzar en su entendimiento. Este aspecto es particularmente importante en países megadiversos como Colombia donde una revisión sobre el estado del arte y especialmente del grado de cobertura del muestreo de un determiando grupo, podría ser una herramienta muy importante, para orientar las acciones de investigación y conservación.

La subfamilia Scarabaeinae (Coleoptera: Scarabaeidae) es un grupo que comprende aproximadamente 5900 especies distribuidas en 250 géneros a nivel mundial (ScarabNet, 2009; Schoolmeesters, 2010; Bouchard et al., 2011), sobresaliendo las regiones tropicales como las más diversas, con ensamblajes complejos, que se simplifican hacia las zonas templadas (Cambefort, 1991a; Gill, 1991; Halffter, 1991). Entre las principales funciones ecológicas de este grupo de insectos se destacan: su contribución al reciclaje de nutrientes, fertilización y aireación del suelo, dispersión secundaria de semillas y control de estados inmaduros de moscas y nemátodos de importancia médica (Nichols et al., 2008). Debido a estas funciones y a la relación con el excremento de vertebrados (Halffter \& Matthews, 1966; Cambefort, 1991b), los escarabajos coprófagos han sido propuestos como eficientes bioindicadores del grado de perturbación ambiental en sistemas naturales (Halffter \& Favila, 1993; McGeoch et al., 2002; Escobar, 2004; Spector, 2006; Nichols et al., 2007; Noriega et al., 2007a; Gardner et al., 2008).

Para el caso de Colombia se registran 305 especies de escarabajos coprófagos agrupadas en 40 géneros. El conocimiento de la riqueza, distribución e historia natural de este grupo ha aumentado significativamente en las últimas décadas a raíz del incremento de estudios en diferentes regiones del país (Howden \& Campbell, 1974; Howden \& Nealis, 1975; Medina et al., 1990; Pardo \& Rubiano, 1994; Pardo et al., 1995; Martín-Piera \& Fernández-Torres, 1996; Medina \& Kattan, 1996; Pardo-Locarno \& Henao, 1996; Amat et al., 1997; Escobar, 1997; Pardo, 1997; Álvarez et al., 1999; Amézquita et al., 1999; Castellanos et al., 1999; Escobar, 2000a; Escobar \& Chacón de Ulloa, 2000; Gasca \& Ospina, 2000; GEMA, 2002; Laverde et al., 2002; Medina et al., 2002; Noriega, 2002a; Pardo \& Castillo, 2002a; 2002b; Bustos-Gómez \& Lopera, 2003; Escobar, 2003a;, 2003b; Huertas et al., 2003; Neita et al., 2003; Pulido et al., 2003; Escobar, 2004; García \& Pardo-Locarno, 2004; Noriega, 2004; Pardo et al., 2004; Escobar et al., 2005; Gasca, 2005; Escobar et al., 2006; Fuentes \& Camero, 2006; Molano \& Morales, 2006; Pulido-H, 2006; Arango et al., 2007; Escobar et al., 2007; Esparza \& Amat, 2007; Giraldo, 2007; Molina, 2007; Noriega et al., 2007a; 2007b; Padilla-Gil \& Halffter, 2007; Pardo-Locarno, 2007; Quintero et al., 2007; Jiménez et al., 2008; Noriega \& Botero-Trujillo, 2008; Noriega \& Calle, 2008; Noriega et al., 2008a; Orozco \& Pérez, 2008; Arango \& Montes, 2009; Bohórquez \& Montoya, 2009; Higuera-Díaz \& OspinaCorrea, 2009; Martínez et al., 2009; Murgueitio \& Giraldo, 2009; Barraza et al., 2010; ConchaLozada et al., 2010; Giraldo \& Murgueitio, 2010; Girón-Vanderhuck et al., 2010; Martínez et al., 2010a; 2010b; 2010c; Murillo et al., 2010; Santos-Heredia et al., 2010; Castellanos et al., 2011; Castillo et al., 2011; Giraldo et al., 2011; Neita \& Escobar, 2011; Noriega \& Acosta, 2011; Solis et al., 2011; Cardenas-Bautista et al., 2012; Delgado-Gómez et al., 2012; Noriega, 2012; Noriega et al., 2012a; Blanco et al., 2013; Noriega \& Navarrete-Heredia, 2013; Otavo et al., 2013).

También se han realizado algunos registros de géneros y especies, descripciones de nuevas especies, revisiones y claves taxonómicas que han mejorado el nivel de identificación de este 
grupo en Colombia (Medina \& Lopera, 2000; Vítolo, 2000a; Noriega, 2002b; Vítolo, 2004; Noriega et al., 2006; Pulido et al., 2007; Rivera \& Wolff, 2007; Noriega et al., 2008b; González et al., 2009; Navarro et al., 2009; SarmientoGarcés \& Amat-García, 2009; Noriega et al., 2009; Camero, 2010; Molano \& Medina, 2010; Arias-Buriticá et al., 2011; Noriega et al., 2011; Cultid \& Giraldo, 2012; Noriega et al., 2012b; Arias-Buriticá \& Vaz-De-Mello, 2013; Cupello \& Vaz-De-Mello, 2013; Arias \& Medina 2014). Igualmente la publicación de algunas sinopsis regionales y nacionales, así como guías de campo, métodos de estudio y manuales de monitoreo han contribuido a acumular un significativo acervo de información, que permite un mejor entendimiento de los patrones biogeográficos de este grupo (Escobar \& Medina, 1996; Escobar, 2000b; Medina et al., 2001; Amat \& Trujillo, 2004; Noriega et al., 2007c; Pérez \& Erazo, 2008; Medina \& Pulido, 2009; Noriega, 2009; Noriega \& Fagua, 2009; Camero \& Lobo, 2010; Cultid et al., 2012; MartínezQuintero et al., 2013).

Sin embargo, a pesar de este importante esfuerzo, continúan existiendo en la actualidad muchos trabajos de pregrado y postgrado que aún no se han publicado en su totalidad, lo cual dificulta no solo su consulta sino la articulación de una base de datos completa a nivel nacional (Escobar, 1994; Lopera, 1996; Delgado, 1998; Quintero, 1998; Camacho, 1999; Hernández \& Elejalde, 2000; Vítolo, 2000b; Bustos, 2001; Castro, 2001; Noriega, 2001; Narváez, 2002; Torres, 2002; Bohórquez, 2003; Castaño, 2003; Garzón et al., 2003; Pérez, 2003; Fuentes, 2004; Motta \& Loáiza, 2004; Posada, 2004; Rivera, 2004; Ardila, 2005; Bernal, 2005; Garcia \& Ospino, 2005; Giraldo et al., 2005; Herrera, 2005; Herrera \& Gutiérrez, 2005; Jiménez \& Mendieta, 2005; Molina, 2005; Solis, 2005; Castillo \& Osorio, 2006; Esparza, 2006; Cultid, 2007; Acevedo \& Aguas, 2007; Palacio, 2007; Santos, 2007; Alvarado, 2008; Arias, 2008; Barón, 2008; Bedoya, 2008; Casas \& Pineda, 2008; González, 2008; Murcia, 2008; Noriega, 2008; Olarte, 2008; Rodríguez, 2008; Rosado, 2008; Ruíz, 2008; Escobar, 2009; Barraza \&
Montes, 2009; Martínez, 2009; Moreno, 2009; Murillo, 2009; Navarro \& Roman, 2009; Espitia, 2010; López, 2010; Montes, 2010; Otavo, 2010; Salcedo \& Sierra, 2010; Ardila, 2011; Arias, 2011; Cardenas, 2011; Hernández, 2011; López \& Martínez, 2011; Moreno-Pérez, 2011; Redondo \& Herazo, 2011; Vargas \& Velandia, 2011; Villada-Bedoya, 2011; Agudelo, 2012; Amell, 2012; Botina \& Castillo, 2012; Delgado-Gómez, 2012; González \& Copete, 2012; Pineda \& Vanegas, 2012; Uribe, 2012; Castillo, 2013; Vargas-Pérez, 2013). Adicionalmente, se registra una gran cantidad de trabajos presentados en congresos y/o simposios a nivel nacional e internacional, donde solo existe un corto resumen en las memorias, los cuales carecen de información básica y son de limitado acceso, razón por lo cual no fueron incluidos en esta revisión.

Debido a lo anterior, el propósito de este documento es hacer una revisión de los trabajos publicados, trabajos de pregrado, postgrado e informes institucionales, así como una revisión de las colecciones existentes en el país, que permita construir una línea base sobre el grado de cobertura del muestreo de escarabajos coprófagos en Colombia. Este análisis permitirá identificar áreas prioritarias de estudio, con miras a focalizar esfuerzos que complementen los inventarios a nivel nacional. También se presenta información sobre las publicaciones a la fecha, líneas de investigación y un análisis a diferentes escalas espaciales (localidades, departamentos, ecosistemas y regiones biogeográficas) en el país.

\section{MATERIALES Y MÉTODOS}

Recopilación y análisis de la información: Se realizó una revisión exhaustiva de la bibliografía existente, utilizando 12 bases de datos referenciales (Catálogo Biblioteca Luis Ángel Arango, Catálogo Biblioteca Virtual de Antioquia, DialNet, ISI Web of Science, Latindex, PLoS, ProQuest, PubMed, REDALYC, Scopus, Science Direct y SciELO), seleccionando todos los artículos publicados, trabajos de pregrado, postgrado e 
informes institucionales realizados en Colombia. Adicionalmente, se revisó la información consignada en los especímenes depositados en 26 colecciones entomológicas a nivel nacional (Cuadro 1 y Cuadro 2). A cada uno de los ejemplares registrados se le tomaron los datos de las coordenadas geográficas donde fueron capturados, generando con esta información una matríz de todas las localidades muestreadas. Posteriormente, se realizó una cuadrícula sobre el territorio Colombiano de $0.5 \times 0.5$ grados (Morrone, 1994; Crisci et al., 1999), asignando a cada cuadrícula las localidades muestreadas, según los datos de georeferenciación.

Los mapas y el análisis de la cobertura se realizaron con el programa ArcGis v. 9.3 a una escala de 1:100000 con respecto a las planchas del Instituto Geográfico "Agustín Codazzi” de Colombia (IGAC, 2013). Los puntos obtenidos a partir de las localidades georeferencidas se digitalizaron en formato vectorial, asociando a cada punto de muestreo una circunferencia con radio de $10 \mathrm{~km}$ (área $=314.16 \mathrm{~km}^{2}$ ). Se sumó el área de todas las localidades muestreadas, estimando el porcentaje de cobertura, con relación al área total de Colombia (área total - área muestreada del territorio colombiano). Para el cálculo de la cobertura a nivel regional y nacional se utilizaron todos los registros encontrados, pero para los análisis a nivel de localidades, departamentos y ecosistemas se utilizaron únicamente los trabajos publicados, con el objetivo de facilitar la consulta de la información por parte de los lectores.

\section{RESULTADOS}

Se revisaron 16940 ejemplares, de los cuales 10562 tenían etiquetas con alguna información corológica o de recolecta que permitió conocer o inferir su ubicación geográfica. Se encontraron registros para 232 especies, cuyas identificaciones estaban confirmadas por especialistas (solo el $27 \%$ del material examinado estaba correctamente identificado a nivel de especie, Cuadro 1, Noriega, 2008). Según la información bibliográfica y de colecciones, se registran 386 localidades muestreadas en el territorio colombiano (Fig. 1), aclarando que todas las localidades que aparecen en el mapa no poseen el mismo esfuerzo de muestreo, por lo que un punto puede representar desde una recolecta ocasional utilizando una única trampa, hasta estudios con varios transectos en diferentes épocas y/o años. Las localidades registradas en las colecciones examinadas van desde 1917 hasta 2005 (no se incluyen datos posteriores ya que la revisión de las colecciones se inicio ese año), reportándose un significativo incremento (más del 200\%) en los últimos años en el número de localidades muestreadas (Fig. 2).

Publicaciones de escarabajos coprófagos a nivel nacional: A nivel nacional existe una gran proporción de trabajos de pregrado y postgrado que aún no han sido publicados $(\mathrm{n}=76,40 \%$, Fig. 3a), de los cuales la mayoría

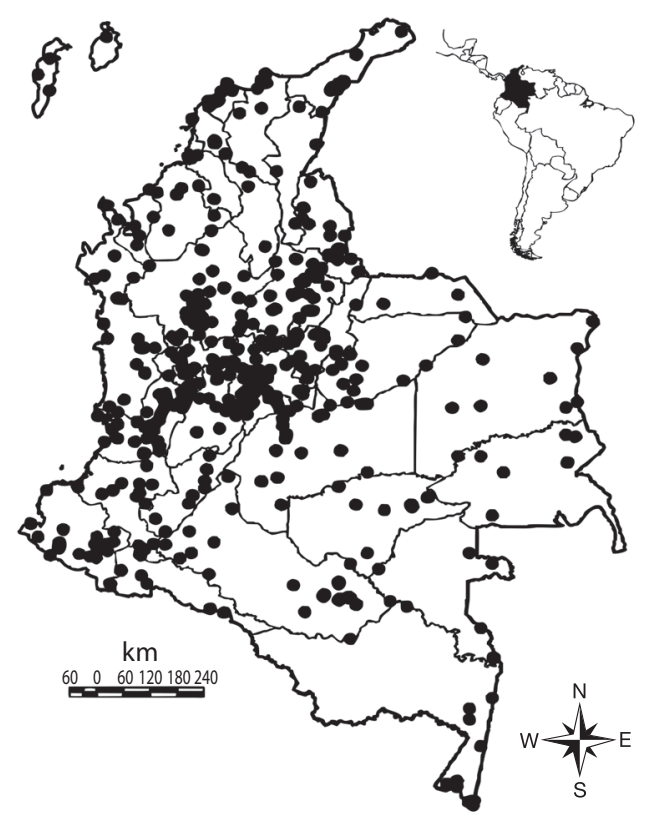

Fig. 1. Distribución de las localidades para Colombia con reportes de escarabajos coprófagos según literatura (1974-2014) y especímenes registrados en las colecciones (1917-2005).

Fig. 1. Distribution of localities in Colombia with reports for dung beetles, using literature (1974-2014) and register specimens from collections (1917-2005). 


\section{CUADRO 1}

Número de especies registradas por género de la subfamilia Scarabaeinae para Colombia

TABLE 1

Number of species registered by genus of the subfamily Scarabaeinae for Colombia

\begin{tabular}{|c|c|c|}
\hline Tribu & Género & spp. \\
\hline \multirow[t]{4}{*}{ Ateuchini } & Agamopus Bates, 1887 & 1 \\
\hline & Anomiopus Westwood, 1842 & 1 \\
\hline & Ateuchus Weber, 1801 & 7 \\
\hline & Uroxys Westwood, 1842 & 15 \\
\hline \multirow[t]{7}{*}{ Demarziellini } & Bdelyrus Harold, 1869 & 8 \\
\hline & Deltorhinum Harold, 867 & 1 \\
\hline & Eutrichillum Martínez, 1968 & 2 \\
\hline & Genieridium Vaz-De-Mello, 2003 & 2 \\
\hline & Scatimus Erichson, 1847 & 3 \\
\hline & Trichillum Harold, 1868 & 1 \\
\hline & Trichillidium Vaz-De-Mello, 2008 & 1 \\
\hline \multirow[t]{12}{*}{ Deltochilini } & Anisocanthon Martínez \& Pereira, 1956 & 2 \\
\hline & Canthon Hoffmannsegg, 1817 & 36 \\
\hline & Canthonella Chapin, 1930 & 1 \\
\hline & Cryptocanthon Balthasar, 1942 & 12 \\
\hline & Deltochilum Eschscholtz, 1822 & 21 \\
\hline & Hansreia Halffter \& Martínez, 1977 & 1 \\
\hline & Malagoniella Martínez, 1961 & 1 \\
\hline & Pseudocanthon Bates, 1887 & 2 \\
\hline & Scatonomus Erichson, 1835 & 1 \\
\hline & Scybalocanthon Martínez, 1948 & 8 \\
\hline & Sylvicanthon Halffter \& Martínez, 1977 & 2 \\
\hline & Sinapisoma Boucomont, 1928 & 1 \\
\hline \multirow[t]{5}{*}{ Coprini } & Canthidium Erichson, 1847 & 27 \\
\hline & Copris Muller, 1764 & 2 \\
\hline & Dichotomius Hope, 1838 & 36 \\
\hline & Homocopris Burmeister, 1846 & 1 \\
\hline & Ontherus Erichson, 1847 & 18 \\
\hline Oniticellini & Eurysternus Dalman, 1824 & 18 \\
\hline \multirow[t]{2}{*}{ Onthophagini } & Digitonthophagus Balthasar, 1959 & 1 \\
\hline & Onthophagus Latreille, 1802 & 31 \\
\hline \multirow[t]{9}{*}{ Phanaeini } & Coprophanaeus Olsoufieff, 1924 & 9 \\
\hline & Dendropaemon Perty, 1830 & 3 \\
\hline & Diabroctis Gistel, 1857 & 2 \\
\hline & Gromphas Brullé, 1834 & 3 \\
\hline & Oruscatus Bates, 1870 & 1 \\
\hline & Oxysternon Laporte-Castelnau, 1840 & 7 \\
\hline & Phanaeus MacLeay, 1819 & 8 \\
\hline & Sulcophanaeus Olsoufieff, 1924 & 7 \\
\hline & Tetramereia Klages, 1907 & 1 \\
\hline Total & 40 & 305 \\
\hline
\end{tabular}

Datos basados en material de colecciones y literatura. Data based on material of collections and literature:

Escobar \& Medina, 1996; Escobar, 2000b; Medina et al., 2001; Noriega, 2002b; Noriega et al., 2007a; Pulido et al., 2007; Noriega et al., 2008a; Vaz-De-Mello, 2008; González et al., 2009; Medina \& Pulido, 2009; Camero, 2010; Molano \& Medina, 2010; Vaz-De-Mello et al., 2010; Cupello \& Vaz-De-Mello, 2013; Arias \& Medina, 2014. 
CUADRO 2

Colecciones nacionales incluidas en el análisis donde se encontraron especímenes de la subfamilia Scarabaeinae para Colombia

TABLE 2

National collections included in the analysis where specimens of the subfamily Scarabaeinae for Colombia were examined

\begin{tabular}{|c|c|c|}
\hline Acrónimo & Nombre Colección & Ciudad \\
\hline CCM-FM & Colección Personal Claudia Medina-Fredy Molano. & Tunja \\
\hline CECC & Colección Escarabajos Coprófagos de Colombia. & Bogotá \\
\hline CEUA & Colección Entomológica Universidad de Antioquia. & Medellín \\
\hline CEUC & Colección Entomológica Universidad de Córdoba. & Montería \\
\hline CEUM & Colección Universidad del Magdalena. & Santa Marta \\
\hline CEUNP & Colección Entomológica Universidad Nacional Sede Palmira. & Palmira \\
\hline CFPL & Colección Familia Pardo Locarno. & Palmira \\
\hline CJAN & Colección de Referencia Jorge Ari Noriega. & Bogotá \\
\hline CROO & $\begin{array}{l}\text { Colección de Referencia Oscar Ortega Museo Francisco Luis Gallego Universidad } \\
\text { Nacional sede Medellín. }\end{array}$ & Medellín \\
\hline $\mathrm{IAvH}$ & Instituto Alexander von Humboldt. & Villa de Leyva \\
\hline ICN-MHN & $\begin{array}{l}\text { Colección de Zoología Museo de Historia Natural Universidad Nacional de } \\
\text { Colombia. }\end{array}$ & Bogotá \\
\hline IMCN & Colección Zoológica de Referencia Científica INCIBA. & Cali \\
\hline MEFLG & Museo Entomológico Francisco Luis Gallego Universidad Nacional sede Medellín. & Medellín \\
\hline MEPB & Museo Entomológico Piedras Blancas Santa Elena. & Medellín \\
\hline MHN-UC & Museo de Historia Natural Universidad del Cauca. & Popayán \\
\hline MHNUPN & Museo de Historia Natural Universidad Pedagógica Nacional. & Bogotá \\
\hline MLS & Museo de la Salle Universidad de La Salle. & Bogotá \\
\hline MPUJ & Museo Javeriano de Historia Natural Lorenzo Uribe s.j. & Bogotá \\
\hline MUA & Colección de Ciencias Naturales Universidad de Antioquia. & Medellín \\
\hline MUSENUV & Museo de Entomología de la Universidad del Valle. & Cali \\
\hline PSO & Colección Zoológica Universidad de Nariño. & Pasto \\
\hline UARC & Universidad del Atlántico Región Caribe UARC. & Barranquilla \\
\hline UIS & Museo de Historia Natural Universidad Industrial de Santander. & Bucaramanga \\
\hline UNAB & Museo Entomológico Facultad de Agronomía Universidad Nacional de Colombia. & Bogotá \\
\hline UPTC & $\begin{array}{l}\text { Museo de Historia Natural Luis Gonzalo Andrade Universidad Pedagógica y } \\
\text { Tecnológica de Colombia. }\end{array}$ & Tunja \\
\hline ZOOLOGIA & Colección de Zoología General. & Pamplona \\
\hline
\end{tabular}

corresponden a estudios ecológicos (85\%) y una baja proporción a trabajos taxonómicos (8\%, Fig. 3b). De los trabajos publicados la mayoría corresponden a estudios ecológicos $(\mathrm{n}=87,77 \%)$ y una menor proporción a trabajos taxonómicos, listados de especies y registros puntuales $(n=26,23 \%)$. En este sentido, son muy pocos los artículos de escarabajos coprófagos a nivel nacional que se encuentran publicados en revistas internacionales (Cuadro 3). Una de las temáticas menos estudiadas son los trabajos de comportamiento, ciclos de vida $\mathrm{y}$ 


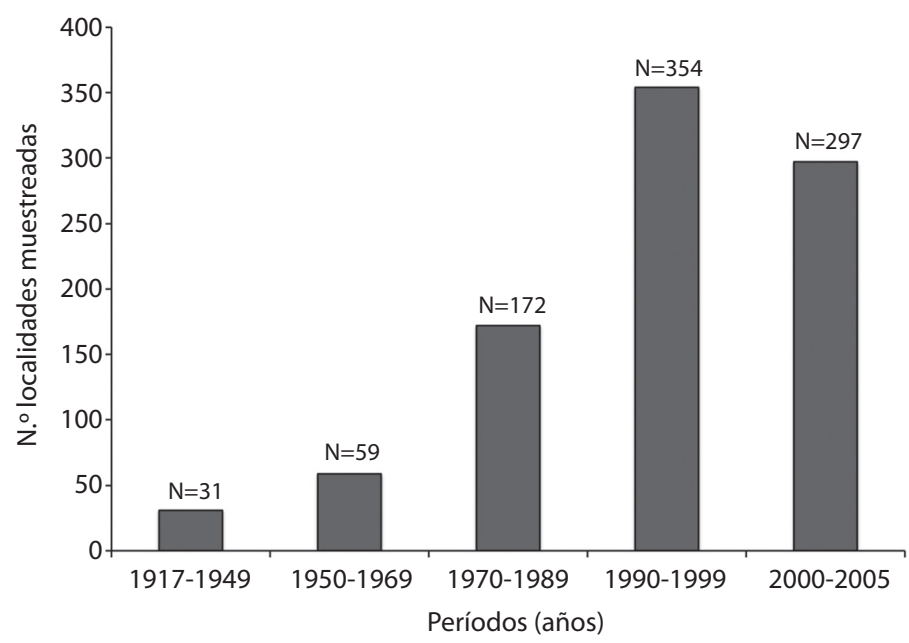

Fig. 2. Número de localidades muestreadas para escarabajos coprófagos en Colombia según material de colecciones en cinco periodos: 1917 a 1949, de 1950 a 1969, de 1970 a 1989 , de 1990 a 1999 y del 2000 al 2005.

Fig. 2. Number of sampling localities for dung beetles in Colombia using specimens of collections in five periods: 1917 to 1949, 1950 to 1969,1970 to 1989,1990 to 1999 and 2000 to 2005 .

fisiología. De igual manera, al momento de realizar este análisis, no se registra para Colombia

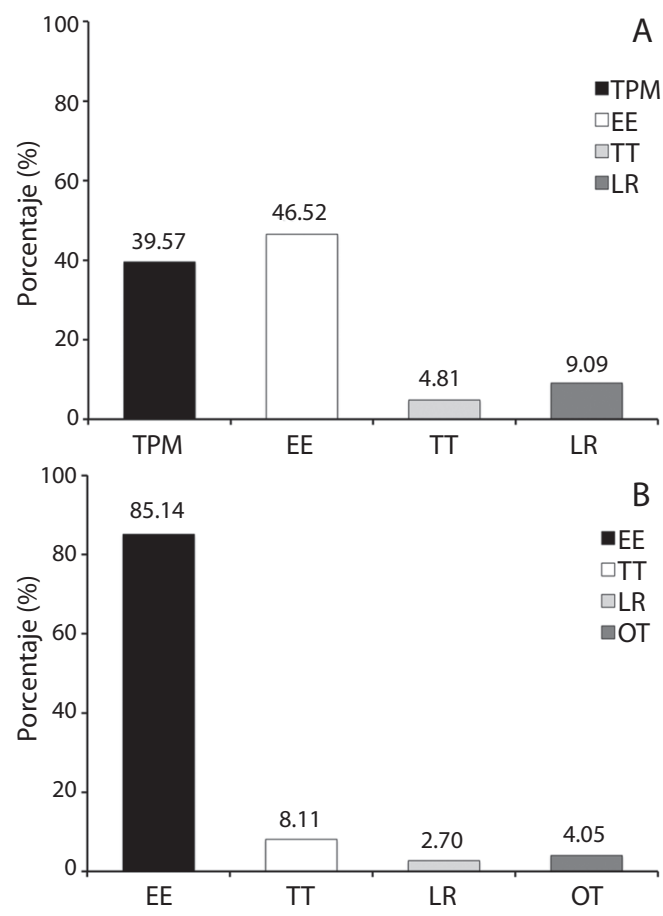

ningún estudio en biología molecular con este grupo.

Al realizar un análisis histórico se evidencia un importante incremento en el número de trabajos de investigacion en los últimos años, en más del $200 \%$ en el periodo $2000-2005$ y en más del $200 \%$ en el periodo 2006-2014 (Fig. 4), coincidiendo con lo observado a nivel nacional (Arbeláez-Cortés, 2013). Con respecto a las temáticas, el principal incremento se atribuye a los trabajos de pregrado y postgrado

Fig. 3. Proporción (\%) de trabajos de escarabajos coprófagos en Colombia según literatura analizada (1974-2014). A) Trabajos de pregrado y postgrado aun no publicados (TPM) y trabajos ya publicados en tres categorías (EE: estudios ecológicos, TT: trabajos taxonómicos y LR: listados y registros). B). Categorías de los trabajos de pregrado y postgrado no publicados (convenciones iguales que A, OT: Otros temas).

Fig. 3. Proportion (\%) of dung beetles studies in Colombia according to analyzed literature (1974-2014). A) Undergraduate and postgraduate studies not yet published (TPM) and studies already published in three categories (EE: ecological studies, TT: taxonomic works and LR: listings and registries). B). Categories of undergraduate and postgraduate studies not published (same conventions as A, OT: Other subjects). 


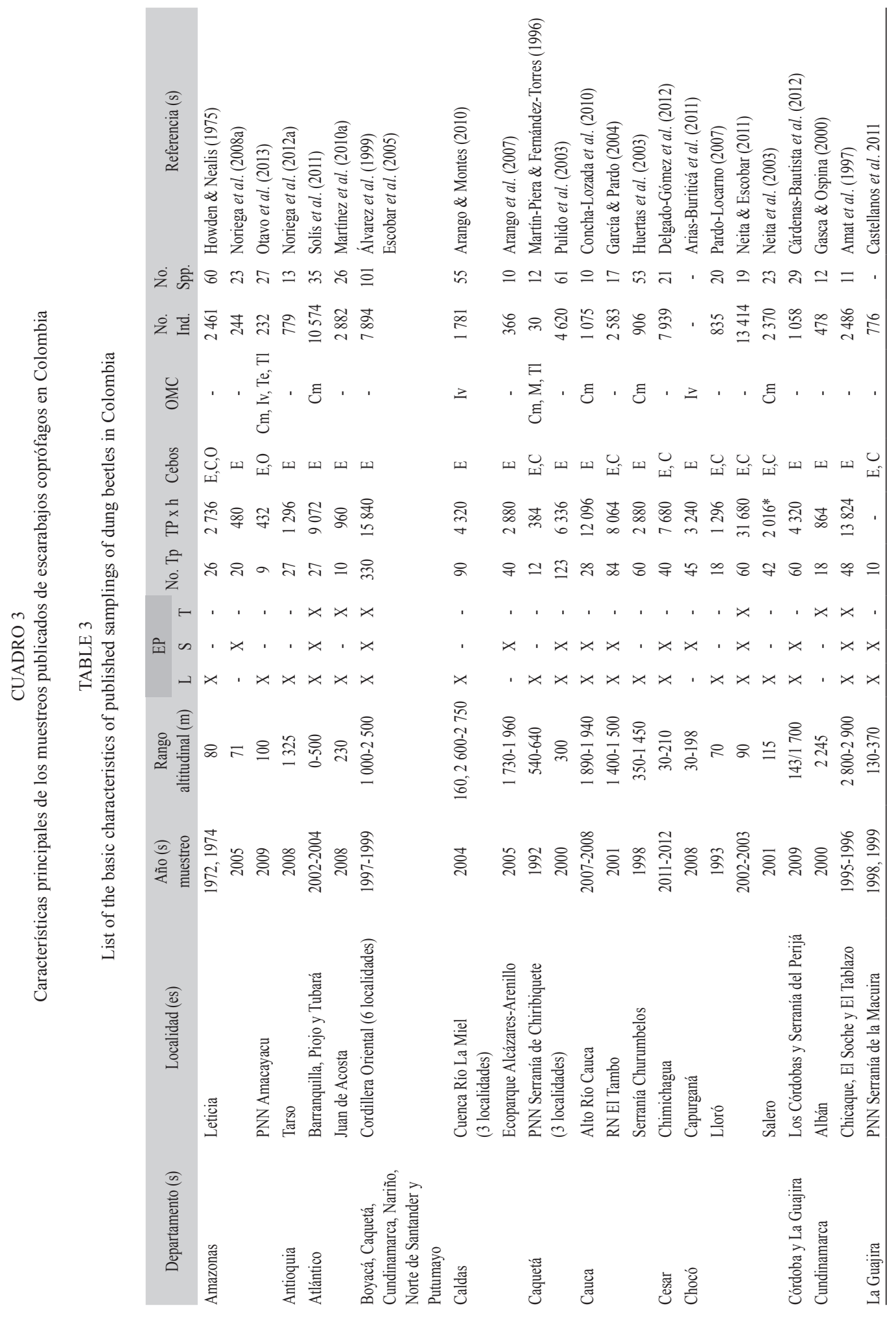




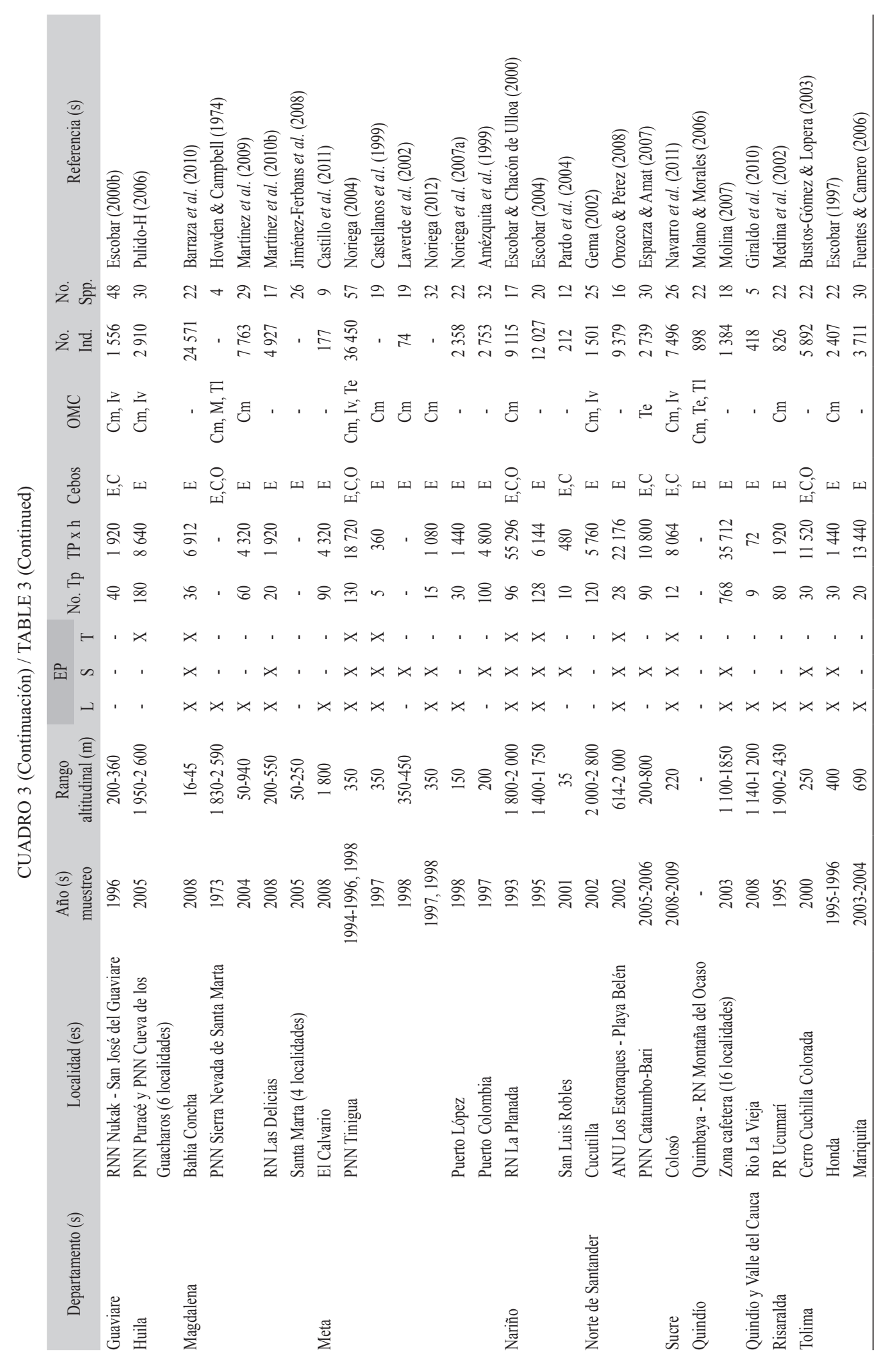

(c) (i) $\ominus$ Rev. Biol. Trop. (Int. J. Trop. Biol. ISSN-0034-7744) Vol. 63 (1): 97-125, March 2015 


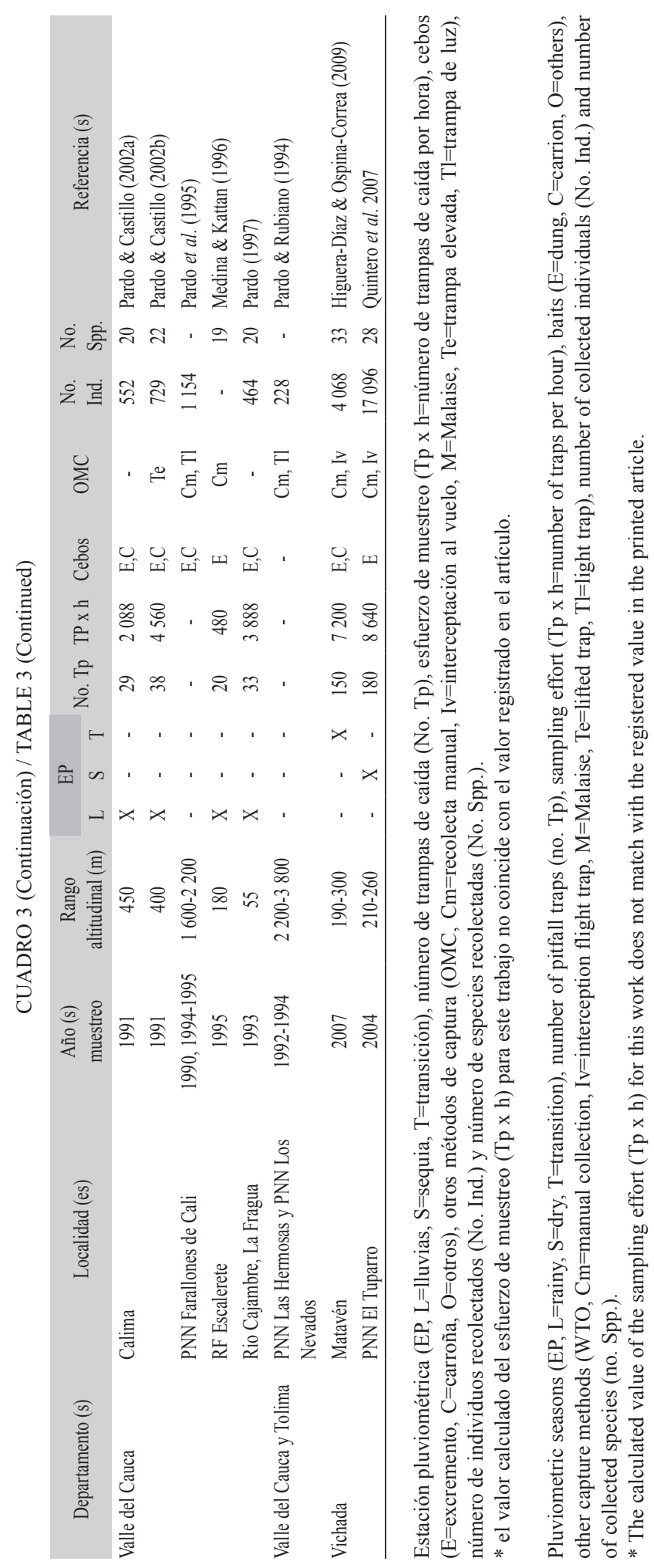




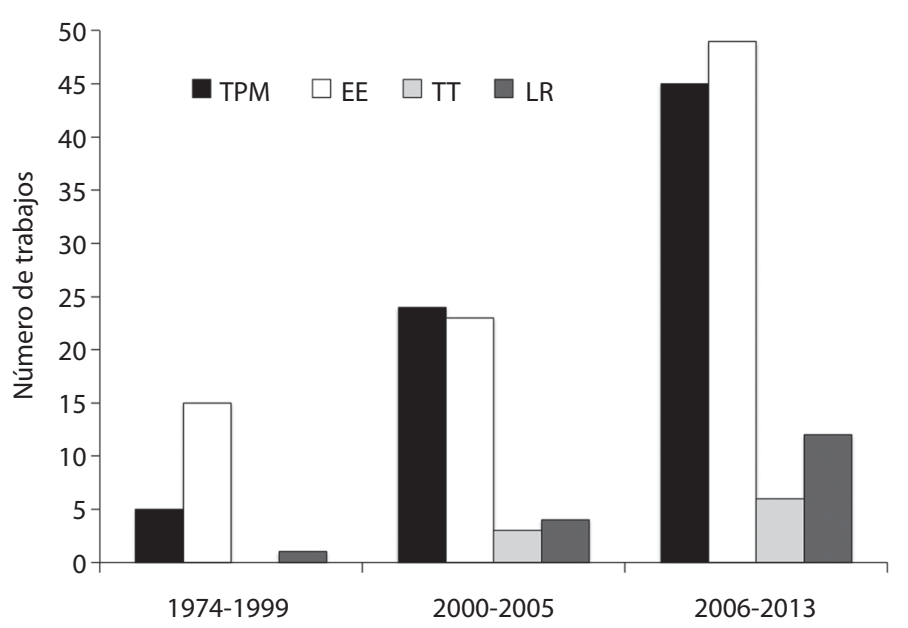

Fig. 4. Número de trabajos de escarabajos coprófagos en Colombia según literatura analizada en tres periodos: 1974 a 1999 , del 2000 al 2005 y del 2006 al 2014 en cuatro categorías: Trabajos de pregrado y postgrado no publicados (TPM), estudios ecológicos publicados (EE), trabajos taxonómicos publicados (TT) y listados y registros publicados (LR).

Fig. 4. Number of dung beetles studies in Colombia according to literature in three periods: 1974 to 1999, from 2000 to 2005 and from 2006 to 2014 in four categories: Undergraduate and postgraduate studies not published (TPM), ecological studies (EE), taxonomic works published (TT) and published listings and registries (LR).

no publicados y a los estudios ecológicos publicados.

Cobertura a nivel de las localidades mejor muestreadas con publicaciones: $\mathrm{Al}$ analizar la cobertura a nivel nacional utilizando las cuadriculas de $0.5 \times 0.5$ grados se evidencia que existen zonas mucho mejor muestreadas en la región Andina que en las demás regiones (Fig. 5a). A partir del análisis detallado de las publicaciones, existe un total de 64 localidades en Colombia (16.58\%), en donde parte o la totalidad de la información obtenida aparece en algún artículo científico ( $\mathrm{n}=60$, Cuadro 3, Fig. 5b).

Las localidades que presentan el mayor esfuerzo de muestreo (Tp: número de trampas de caída x h: horas de muestreo, Tpxh) son: RN La Planada (Reserva Natural - Nariño, Tpxh=61 440), Lloró (Chocó, Tpxh=32 976), ANU Los Estoraques (Área Natural Única - Norte de Santander, Tpxh=22 176), PNN Tinigua (Parque Nacional Natural - Meta, $\mathrm{Tpxh}=20$ 160) y Mariquita (Tolima, Tpxh=13 440) (Cuadro 4). Al realizar una correlación entre los esfuerzos de muestreo y las abundancias se encontró una relación del $43 \%\left(r^{2}=0.43\right.$, $\mathrm{p}<0.05, \alpha=0.05)$, mientras que entre el esfuerzo de muestreo y la riqueza la relación es de tan solo el $6 \%\left(r^{2}=0.06, \mathrm{p}<0.05, \alpha=0.05\right)$.

\section{Cobertura a nivel de los departamentos:} Encontramos registros ( $\mathrm{n}=$ número de localidades muestreadas) para todos los departamentos del país, sin embargo solo algunos departamentos que contienen grandes centros urbanos tienen un número alto de localidades muestreadas como: Cundinamarca $(\mathrm{n}=35)$, Antioquia $(n=33)$, Valle del Cauca $(n=25)$ y Boyacá $(\mathrm{n}=23)$ (Fig. 1). Otro grupo de departamentos entre los cuales están Chocó $(\mathrm{n}=21)$, Santander $(\mathrm{n}=20)$, Meta $(\mathrm{n}=19)$, Caquetá $(\mathrm{n}=17)$, Nariño $(n=16)$, Tolima $(n=15)$ y Casanare $(n=14)$ están en una condición intermedia-alta, con un número medio de puntos de muestreo. Otros departamentos como Norte de Santander $(\mathrm{n}=13)$, Cauca $(\mathrm{n}=13)$, Amazonas $(\mathrm{n}=12)$, Caldas $(n=10)$, Bolivar $(n=8)$, Huila $(n=8)$, Vichada $(n=8)$, Magdalena $(n=7)$, Guainía $(n=7)$, Guaviare $(\mathrm{n}=7)$ y Quindío $(\mathrm{n}=7)$ presentan un 
A

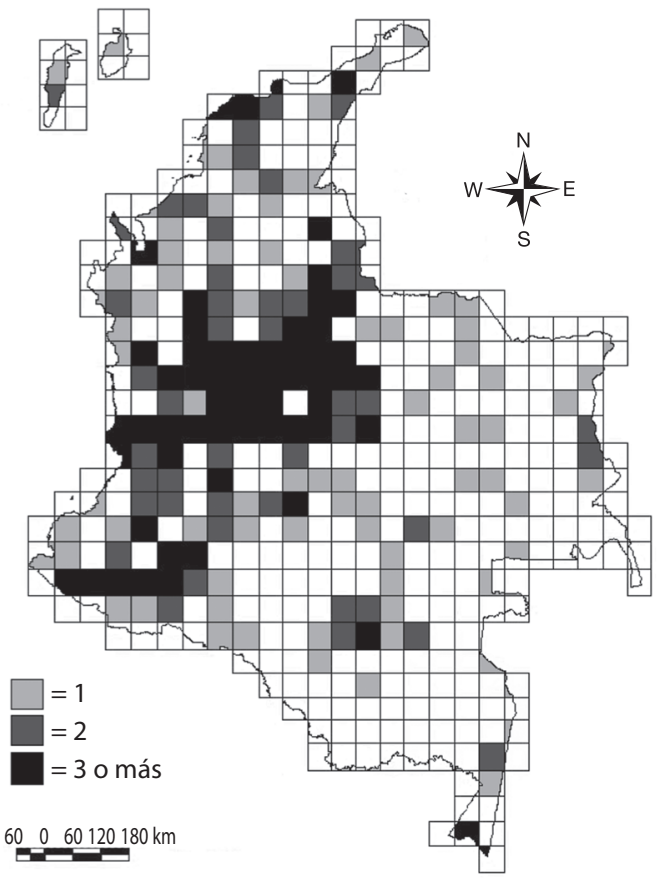

B

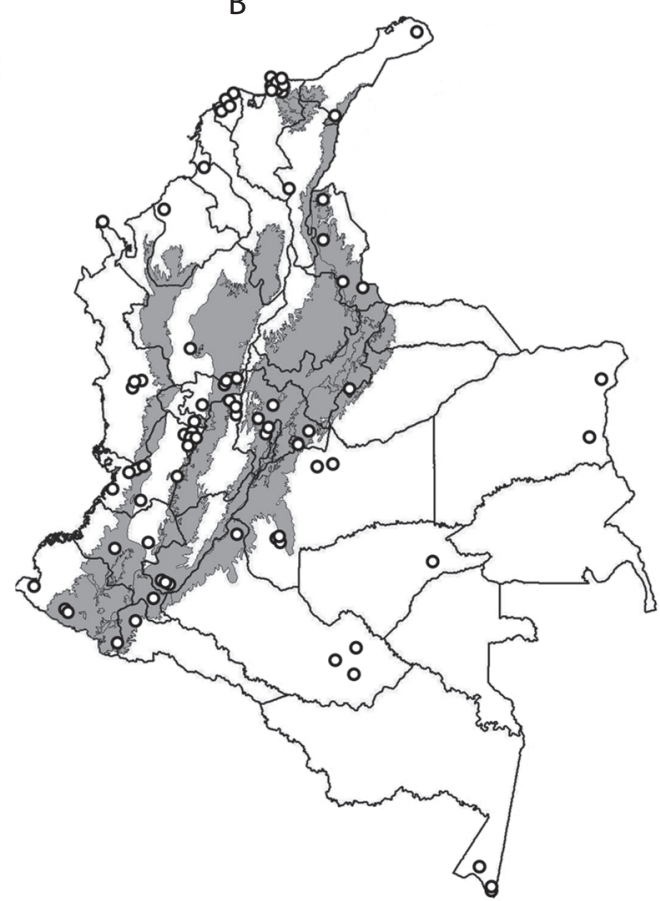

Fig. 5. A) Cuadricula de $0.5 \times 0.5$ grados de las zonas con localidades muestreadas para escarabajos coprófagos (con $1,2,3$ o más localidades), según literatura y especímenes registrados en colecciones para Colombia. B) Localidades que tienen por lo menos un artículo publicado de escarabajos coprófagos para Colombia (1974-2014, según Tabla 3).

Fig. 5. A) Squares of $0.5 \times 0.5$ degrees of zones with localities sampled for dung beetles (with $1,2,3$ or more localities), according to literature and specimens registered in collections for Colombia. B) Localities that at least have a published article of dung beetles for Colombia (1974-2014, according to Table 3).

\section{CUADRO 4}

Las cinco localidades mejor muestreadas con trabajos publicados de escarabajos coprófagos en Colombia según el esfuerzo de muestreo

TABLE 4

The five better-sampled localities with published studies for dung beetles in Colombia according to the sampling effort

\begin{tabular}{|c|c|c|c|c|c|c|c|}
\hline Departamento & Localidad & Año (s) muestreo & Altura (m) & No. Tp & $\mathrm{Tp} \times \mathrm{h}$ & No. Spp. & Referencia (s) \\
\hline Nariño & RN La Planada & 1993,1995 & $1400-2000$ & 224 & 61440 & 21 & $\begin{array}{l}\text { Escobar \& Chacón de Ulloa } \\
(2000) \\
\text { Escobar (2004) }\end{array}$ \\
\hline Choco & Lloró & $1993,2002-2003$ & $70-90$ & 78 & 32976 & 24 & $\begin{array}{l}\text { Pardo-Locarno (2007) } \\
\text { Neita \& Escobar (2011) }\end{array}$ \\
\hline Norte de Santander & ANU Los Estoraques & 2002 & $614-2000$ & 28 & 22176 & 16 & Orozco \& Pérez (2008) \\
\hline Meta & PNN Tinigua & 1994-1996, 1997 & $350-450$ & 135 & 20160 & 62 & $\begin{array}{l}\text { Castellanos et al. (1999) } \\
\text { Laverde et al. (2002) } \\
\text { Noriega (2004) } \\
\text { Noriega (2012) }\end{array}$ \\
\hline Tolima & Mariquita & 2003-2004 & 690 & 20 & 13440 & 30 & Fuentes \& Camero (2006) \\
\hline
\end{tabular}

Número de trampas de caída (No. Tp), esfuerzo de muestreo ( $\mathrm{Tp}$ x h=número de trampas de caída por hora) y número de especies (No. Spp.).

Number of pitfall traps (No. Tp), sampling effort ( $\mathrm{Tp} x \mathrm{~h}=$ number of traps per hour) and number of species (no. Spp.). 
nivel de muestreo intermedio-bajo, con pocas localidades y con extensas áreas sin ningún registro. Finalmente, existen algunos departamentos como Córdoba $(n=6)$, La Guajira $(n=6)$, Putumayo ( $\mathrm{n}=6)$, Risaralda $(\mathrm{n}=6)$, Atlántico $(\mathrm{n}=5)$, Vaupés $(\mathrm{n}=5)$, San Andrés y Providencia $(n=4)$, Arauca $(n=3)$, Cesar $(n=3)$ y Sucre $(n=3)$ que están en una situación crítica, al tener muy pocas localidades muestreadas.

A nivel de las publicaciones científicas, los departamentos con un mayor número de artículos son el Valle del Cauca y Meta ( $\mathrm{n}=7)$, Magdalena, Nariño y Norte de Santander $(\mathrm{n}=5)$, seguidos por Caquetá, Chocó, Cundinamarca y Tolima $(n=4)$ (Cuadro 3, Fig. 5b). Existen varios departamentos que a pesar de tener varias localidades muestreadas no tienen ningún artículo publicado como son: Arauca, Bolivar, Casanare, Guainía, Santander y Vaupés.

Cobertura a nivel de los principales
ecosistemas con publicaciones: Teniendo
en cuenta la alta diversidad de ecosistemas
en Colombia (Etter, 1993) y los ecosistemas
muestreados en las publicaciones se observa
una fuerte tendencia a estudiar bosques húme-
dos de tierras bajas, premontanos y monta-
nos (Cuadro 5). Los ecosistemas con mayor
número de publicaciones son el bosque húme-
do premontano Andino ( $\mathrm{n}=13)$ y el bosque

CUADRO 5

Ecosistemas estudiados para escarabajos coprófagos en el territorio Colombiano según los trabajos publicados

TABLE 5

List of studied ecosystems for dung beetles in Colombian territory according to published articles

\begin{tabular}{|c|c|}
\hline Ecosistemas & Referencias \\
\hline Bosque altoandino & Amat et al. 1997 \\
\hline Bosque húmedo Amazónico & $\begin{array}{l}\text { Howden \& Nealis 1975, Martín-Piera \& Fernández-Torres 1996, Castellanos et al. 1999, Laverde et al. } \\
\text { 2002, Noriega 2004, Noriega et al. 2008a, Noriega 2012, Otavo et al. } 2013\end{array}$ \\
\hline $\begin{array}{l}\text { Bosque húmedo del Caribe } \\
\text { (tierras bajas, pre y montano) }\end{array}$ & Howden \& Campbell 1974, Martínez et al. 2009, Cardenas-Bautista et al. 2012 \\
\hline Bosque húmedo del Pacífico & $\begin{array}{l}\text { Medina \& Kattan 1996, Pardo 1997, Pardo \& Castillo 2002a,b, Neita et al. 2003, Pardo et al. 2004, } \\
\text { Pardo-Locarno 2007, Arias-Buritica et al. 2011, Neita \& Escobar } 2011\end{array}$ \\
\hline Bosque húmedo premontano Andino & $\begin{array}{l}\text { Escobar \& Chacón de Ulloa 2000, Huertas et al. 2003, Escobar 2004, García \& Pardo 2004, Escobar } \\
\text { et al. 2005, Fuentes \& Camero 2006, Molano \& Morales 2006, Arango et al. 2007, Esparza \& Amat } \\
\text { 2007, Molina 2007, Arango \& Montes 2010, Castillo et al. 2011, Noriega et al. 2012a }\end{array}$ \\
\hline Bosque húmedo montano Andino & $\begin{array}{l}\text { Pardo \& Rubiano 1994, Pardo et al. 1995, Álvarez et al. 1999, Gasca \& Ospina 2000, GEMA 2002, } \\
\text { Medina et al. 2002, Escobar et al. 2005, Pulido 2006, Orozco \& Pérez 2008, Arango \& Montes 2010, } \\
\text { Concha-Lozada et al. } 2010\end{array}$ \\
\hline Bosque de galería de la Orinoquia & Amézquita et al. 1999, Noriega et al. 2007a \\
\hline Bosque seco del Caribe & $\begin{array}{l}\text { Jiménez-Ferbans et al. 2008, Martínez et al. 2009, Barraza et al. 2010, Martínez et al. 2010a, 2010b, } \\
\text { Navarro et al. 2011, Solís et al. 2011, Delgado-Gómez et al. } 2012\end{array}$ \\
\hline Bosque seco región inter Andina & Escobar 1997, Bustos-Gómez \& Lopera 2003 \\
\hline Bosque de tierra firme e inundables & Escobar 2000b, Pulido et al. 2003 \\
\hline Bosque y sabana de planicies & Quintero et al. 2007, Higuera \& Ospina 2009 \\
\hline Bosque subxerofítico premontano & Orozco \& Pérez 2008 \\
\hline Bosque subxerofítico espinoso & Castellanos et al. 2011 \\
\hline $\begin{array}{l}\text { Sistemas agrícolas, agroforestales } \\
\text { y ganaderos }\end{array}$ & $\begin{array}{l}\text { Medina \& Kattan 1996, Medina et al. 2002, Molina 2007, Giraldo et al. 2010, Neita \& Escobar 2011, } \\
\text { Navarro et al. 2011, Noriega et al. 2012a }\end{array}$ \\
\hline Páramos & Pardo \& Rubiano 1994 \\
\hline
\end{tabular}


húmedo montano Andino ( $\mathrm{n}=11)$, seguidos del bosque húmedo del Pacífico ( $\mathrm{n}=9$ ), el bosque seco del Caribe $(n=8)$ y el bosque húmedo amazónico $(\mathrm{n}=8)$.

Cobertura a nivel de las regiones biogeográficas: Para las regiones biogeográficas propuestas por Hernández et al. (1992), se encontró que algunas regiones como la Amazonía, Orinoquía y parte de la región central del Cinturón Árido Pericaribeño presentan pocas localidades muestreadas y grandes espacios vacíos que carecen de registros (Fig. 1). El caso de las áreas insulares (islas continentales y oceánicas) es deficiente, al tener solamente algunos registros ocasionales y aislados (Arrow, 1933; Medina \& Lopera, 2000; Noriega, 2002b; Cultid \& Giraldo, 2012) pero ningún inventario completo de su fauna (Escobar, 2000b).

La región con el mayor porcentaje de cobertura muestreado es la región Andina con el 34.6\% $\left(\mathrm{n}=202,94166 \mathrm{~km}^{2}\right)$, seguida del Chocó-Magdalena con un 30.8\% $(\mathrm{n}=55$, $\left.25639 \mathrm{~km}^{2}\right)$, el Caribe con un $15.5 \%(\mathrm{n}=44$, $\left.20511 \mathrm{~km}^{2}\right)$, la Amazonía con el $6.5 \%(\mathrm{n}=53$, $24707 \mathrm{~km}^{2}$ ) y finalmente la Orinoquía con el $5.3 \%\left(\mathrm{n}=32,14917 \mathrm{~km}^{2}\right)$. Teniendo en cuenta las publicaciones la región Andina es la que presenta el mayor número de artículos $(\mathrm{n}=32)$, seguida de la región Caribe $(n=16)$, la región de la Amazonía ( $\mathrm{n}=10$ ), el Chocó-Magdalena $(n=9)$ y por último la Orinoquía $(n=4)($ Fig. $5 b)$.

\section{Cobertura a nivel del territorio nacio-} nal: Al consolidar la información a nivel nacional se hace evidente que existen zonas mejor estudiadas, especialmente aquellas cercanas a los centros urbanos, lo cual obedece al efecto de la cercanía y a la facilidad de muestreo que tienen las grandes ciudades (Fig. 1, Fig. 2, Fig. 3, Fig. 4 y Fig. 5). La suma de las localidades incluidas en este análisis constituye un área muestreada aproximada de $121265.76 \mathrm{~km}^{2}$, lo cual al contrastarse con la porción terrestre de Colombia (1 $141748 \mathrm{~km}^{2}$ ) genera un porcentaje de cobertura del $10.62 \%$, dejando un $89.38 \%$ del territorio que aun no ha sido muestreado.
Utilizando la cuadricula de $0.5 \times 0.5$ grados, el nivel de cobertura asciende a $41.2 \%(\mathrm{n}=188$ casillas, Fig. 5a), ya que a esta escala muchas áreas sin muestrear aparecen con algún registro.

El caso del Sistema Nacional de áreas protegidas y especialmente los Parques Nacionales Naturales (PNN) llaman fuertemente la atención, ya que a pesar de ser áreas prioritarias y constituirse en la principal estrategia de conservación in situ de la biodiversidad en Colombia (113909.94km², 9.97\% del territorio nacional), no se encuentran bien estudiadas y muchas de ellas no tienen ningún registro. Según lo encontrado en este análisis solo existen 22 publicaciones para 14 (25\%) de las 56 áreas protegidas en todo el territorio nacional: PNN Amacayacu (Amazonas - Otavo et al., 2013), PNN Catatumbo-Bari (Norte de Santander - Esparza \& Amat, 2007), PNN Cueva de Los Guacharos y PNN Puracé (Huila - Pulido-H, 2006), PNN Las Hermosas y PNN Los Nevados (Valle del Cauca y Tolima - Pardo \& Rubiano, 1994), ANU Los Estoraques (Norte de Santander Orozco \& Pérez, 2008), PNN Farallones de Cali (Valle del Cauca - Pardo et al., 1995), RNN Nukak (Guaviare - Escobar, 2000b), PNN Serranía de Chiribiquete (Caquetá - MartinPiera \& Fernández-Torres, 1996; Pulido et al., 2003), PNN Serranía de la Macuira (La Guajira - Castellanos et al., 2011), PNN Sierra Nevada de Santa Marta (Magdalena - Howden \& Campbell, 1974; García \& Ospina, 2005; Noriega et al., 2007b; Martínez et al., 2009), PNN Tinigua (Meta - Castellanos et al., 1999; Laverde et al., 2002; Noriega, 2002a; 2004; Noriega \& Calle, 2008; Noriega \& Acosta, 2011; Noriega, 2012) y PNN Tuparro (Vichada - Quintero et al., 2007) (Cuadro 3).

\section{DISCUSIÓN}

Análisis de las publicaciones a nivel nacional: Un problema que es fundamental señalar, es el grado de visibilidad internacional de la información que se produce en el país. Muchos de los excelentes trabajos de pregrado y postgrado que se realizan a nivel nacional, es frecuente que se queden como 
literatura gris (documentos olvidados en alguna biblioteca regional a los que nadie tiene acceso) o si llegan a publicarse, en el mejor de los casos, casi nunca lo hacen en revistas internacionales. Lamentablemente, la mayoría terminan en revistas locales no indexadas o con factores de impacto muy bajo y con muy baja circulación (Arbeláez-Cortés, 2013). Sin embargo, es importante señalar el incremento en trabajos taxonómicos, listados de especies y registros puntuales, lo cual estaría demostrando un mayor interés en esta área y un creciente número de estudiantes y profesionales trabajando en temas de taxonomía en Colombia, lo cual puede favorecer la consolidación de la información en este grupo.

Análisis de la cobertura - localidades, departamentos y ecosistemas: El análisis de la cobertura evidenció que las localidades muestreadas no tienen el mismo esfuerzo o intensidad de muestreo, no siendo del todo comparables por las grandes diferencias existentes en el número de transectos, distancia entre transectos, número de trampas, distancia entre trampas, tiempo de muestreo, recolectas en diferentes épocas y/o años, diferentes cebos utilizados y tipos de trampas empleadas. Algunas localidades presentan buenos esfuerzos de muestreo, pero la mayoría no han sido inventariadas a fondo, careciendo de monitoreos en diferentes estaciones y años, de la utilización de diferentes tipos de cebos, del uso de diferentes tipos de trampas y de la inclusión de gradientes verticales y altitudinales.

En este sentido, una de las localidades mejor muestreadas que evidencia una de las mayores riquezas en Colombia es la Serranía de la Macarena-Meta (70 spp., J. Noriega datos inéditos), lo cual es una clara consecuencia del continuo trabajo en esta área durante varios años y épocas (1992, 1994-1998, época de lluvias, sequía y transición), del uso de múltiples métodos de captura (trampas de caída o "pitfall", trampas de interceptación de vuelo, trampas elevadas, recolecta directa y otros), cebos (excremento humano, excremento de vertebrados locales, pescado, hongos y frutas) y del muestreo en diferentes hábitats (playas, bosque inundable, bosque maduro) y micro hábitats, demostrando que para tener un muestreo completo, es necesario un trabajo exhaustivo a nivel espacial y temporal, que involucre varias técnicas de muestreo y la totalidad de posibles micro hábitats (Castellanos et al., 1999; Laverde et al., 2002; Noriega, 2002a; Noriega, 2004; Noriega \& Calle, 2008; Noriega \& Acosta, 2011; Noriega, 2012).

De igual manera los departamentos menos muestreados requieren campañas intensivas $\mathrm{y}$ un aumento en el número de localidades, especialmente en aquellas zonas aisladas y lejanas de los centros urbanos. Con respecto a la cobertura a nivel de los ecosistemas, existen varios que no tienen ningún estudio publicado de escarabajos coprófagos como son: los manglares, zonas desérticas, sabanas naturales y morichales. De igual manera existen muy pocos estudios en páramos, en bosques altoandinos, en bosques inundables, en sistemas agroforestales, ganaderos y en sistemas agrícolas específicos.

Análisis de la cobertura - regiones biogeográficas: El análisis de la cobertura a nivel de las regiones biogeográficas evidenció que la región Andina tiene la mayor cantidad de localidades y área muestreada, sin embargo existen algunas zonas específicas como el flanco occidental de la cordillera Occidental y Central, de las que se desconoce su riqueza, lo cual, sumado a la fuerte presión antrópica que se presenta en las laderas de los valles interandinos (Etter \& Wyngaarden, 2000; González et al., 2011) hace de esta zona un lugar prioritario de investigación (Escobar et al., 2005). En este sentido, los estudios de Escobar et al. (2005; 2006; 2007) en el flanco oriental de la cordillera Oriental, que abarcan un completo gradiente altitudinal y latitudinal, han generado una excelente aproximación a la riqueza de esta subregión.

La región de la Orinoquía es la más pobremente muestreada (en número de localidades y en cobertura), seguida de la Amazonía y la región Caribe. El caso de la región Amazónica 
es especialmente complejo, a pesar de haberse realizado un buen número de estudios (Howden \& Nealis, 1975; Quintero, 1998; Castro, 2001; Pulido et al., 2003; Gasca, 2005; Murcia, 2008; Noriega et al., 2008b; Otavo et al., 2013), es muy poca la información publicada si se tiene en cuenta su extensión $\left(379183 \mathrm{~km}^{2}, 32.9 \%\right.$ del territorio nacional). La región circundante a la ciudad de Leticia es la mejor muestreada, pero hacia el interior del departamento del Amazonas son muy pocos los muestreos realizados. A pesar de lo anterior, es una de las regiones con mayor riqueza reportada para los escarabajos coprófagos, pero carece de información acerca del grado de homogeneidad que presenta, en contraste con otros taxa (Cracraft \& Prum, 1988; Amorim, 2001; Garzón-Orduña \& Miranda-Esquivel, 2007).

La región del Cinturón Árido Pericaribeño no presenta una situación tan crítica, ya que en varios muestreos parece evidenciarse que la fauna existente es bastante homogénea y no presenta variaciones marcadas (Bohórquez, 2003; Rivera, 2004; García \& Ospino, 2005; Herrera, 2005; Solís, 2005; Noriega et al., 2007b; Padilla-Gil \& Halffter, 2007; Jiménez et al., 2008; Noriega, 2008; Ruíz, 2008; Martínez et al., 2009; Navarro \& Roman, 2009; Barraza et al., 2010; Martínez et al., 2010a,b,c; Cardenas, 2011; Castellanos et al., 2011; López \& Martínez, 2011; Redondo \& Herazo, 2011; Cárdenas-Bautista et al., 2012; Delgado-Gómez et al., 2012; Navarro et al., 2012), exceptuando el posible caso de algunos sistemas montañosos aislados que requieren muestreos intensivos como: Sierra de La Macuira (La Guajira), Serranía del Perijá (Norte de Santander, Cesar y La Guajira), Serranía de San Jacinto (Bolívar) y Serranía de San Lucas (Bolívar) que pueden constituirse en enclaves de alta endemicidad (Hernández et al., 1992).

De esta región resalta el caso del Macizo de la Sierra Nevada de Santa Marta (SNSM), como un ejemplo del nivel de desconocimiento en el que se encuentran ciertas áreas en el país. Inicialmente, Howden \& Campbell (1974) registraron cuatro géneros para esta región (Canthidium, Eurysternus, Onthophagus y
Uroxys) sin llegar a identificar a especie el material recolectado. Posteriormente, Escobar (2000b) registró cuatro especies: Ontherus lichyi Martínez, 1947, Ontherus sanctaemartae Genier, 1996, Scybalocanthon darlingtoni (Paulian, 1939) y Sulcophanaeus steinheili (Harold, 1875). Estudios mas recientes y muestreos en zonas aledañas (Noriega, 2001; García \& Ospino, 2005; Noriega et al., 2007b; Jiménez et al., 2008; Martínez et al., 2009) consolidaron un listado de 57 especies para esta zona, anotando que el flanco sur oriental de la SNSM se encuentra todavía submuestreado, lo cual demuestra el importante efecto que puede tener el estudio más detallado de áreas no muestreadas.

La región del Chocó-Magdalena biogeográfico con más del $7 \%\left(83170 \mathrm{~km}^{2}\right)$ del territorio nacional, comprende una franja de $1300 \mathrm{~km}^{2}$ (desde Ecuador a Panamá), constituyéndose en una región megadiversa en lo referente a escarabajos coprófagos, pero aún así se desconoce gran parte de su diversidad a pesar del alto número de trabajos realizados (Medina \& Kattan, 1996; Pardo-Locarno \& Henao, 1996; Pardo, 1997; Pardo \& Castillo, 2002a; 2002b; Neita et al., 2003; Amat \& Trujillo, 2004; Pardo et al., 2004; Pardo-Locarno, 2007; Murillo et al., 2010; Arias-Buriticá et al., 2011; Neita \& Escobar, 2011; Vargas \& Velandia, 2011; Cultid \& Giraldo, 2012). Se registran importantes estudios en los siguientes departamentos y cuencas: Chocó (Río Atrato y Sanjuán), Valle del Cauca (Río Calima, Dagua, Bravo, Dovio y Cajambre), Cauca (sector Munchique y Río Tambito) y Nariño (Río Ñambí y sector de Robles), sin embargo, la mayoría de esta información, se encuentra en resúmenes de congresos o seminarios y no se ha publicado en extenso (L.C. Pardo datos inéditos). A nivel general, se evidencian importantes filiaciones entre esta región y el Cinturón Árido Pericaribeño (Urabá, zona alta Córdoba) y las selvas de Panamá y Costa Rica en Centro América (L.C. Pardo datos indeditos). A pesar de todo lo anterior, extensas áreas del norte del Chocó, costa y piedemonte caucano y nariñense, como los zonobiomas y formaciones ecológicas de 
selvas de Mangual, enclaves subxerofíticos y selvas de niebla, paramillos y páramo permanecen aun sin registros formales.

\section{Análisis de la cobertura a nivel nacio-} nal: El análisis de la cobertura a nivel nacional evidencio que existen zonas bien estudiadas, cercanas a los centros urbanos, sin embargo el porcentaje de cobertura total es inferior al $11 \%$, dejando un $89 \%$ del territorio nacional que aun no ha sido muestreado. La utilización de la cuadricula de 0.5 x 0.5 grados para el análisis, aumenta la cobertura a nivel regional, pero es una ilusión óptica peligrosa de asumir, especialmente en zonas como la región Andina, ya que genera la falsa impresión de ser una región muy bien muestreada en su totalidad, lo cual no concuerda con la realidad. Adicionalmente, algunas de las localidades incluidas en el análisis presentan un alto grado de sobrelapamiento, por lo que el porcentaje de cobertura podría ser aún menor que el valor estimado.

A lo anterior debe sumarse el alto nivel de submuestreo y en algunos casos la total inexistencia de registros. La disimilitud en la cobertura, los grandes vacíos de información y las inmensas zonas alteradas que se presentan en Colombia (Etter et al., 2006; 2008; Sánchez et al., 2012), plantean grandes inquietudes. El caso del Sistema Nacional de PNN es crítico, ya que solo existen publicaciones para el $25 \%$ de las áreas protegidas en todo el país.

Es importante reconocer que algunas de las áreas submuestreadas presentan problemas de orden público, lo cual ha afectado la realización de colectas de manera segura en las últimas cinco décadas (Regalado, 2013). Adicionalmente, esta carencia de registros puede atribuirse a la dificultad de acceso, a las condiciones climáticas complejas y a las barreras administrativas a nivel de permisos para hacer investigación (Fernández, 2011). Sin embargo, es importante señalar que muchas de estas áreas con conflictos armados se han mantenido conservadas debido a que han evitado que colonos, empresas particulares y hasta el mismo gobierno realicen actividades que repercutan negativamente, como señala Frédéric Massé: "la guerra ha permitido que muchos ecosistemas permanezcan intactos" (Semana, 2013).

Es necesario ampliar la cobertura en muchas áreas del país, con miras a completar el listado de especies y su distribución, permitiendo construir un panorama más completo de los patrones biogeográficos, especialmente en zonas fuertemente alteradas, teniendo en cuenta la acelerada perdida de cobertura de bosques en el país (Etter \& Wyngaarden, 2000; González et al., 2011). En este sentido, se recomienda priorizar y enfocar los esfuerzos de muestreo en aquellas áreas no muestreadas o submuestreadas con importancia estratégica a nivel de conservación. Este proceso debe ir de la mano con un programa nacional de consolidación de la información taxonómica, que unifique criterios de identificación, mejore el intercambio entre colecciones, agilice los procesos de tramite para la obtención de permisos de recolecta y propicie mecanismos de estímulo para la formación académica de taxónomos (Fernández, 2011).

Aunque existen buenos esfuerzos en esta dirección, como el trabajo realizado por Cultid et al. (2012) para el eje cafetero, hay que ser muy cautelosos con este tipo de acercamientos, ya que las revisiones taxonómicas que se están realizando a nivel nacional, están basadas en un $10.62 \%$ de cobertura, lo cual podría generar una visión simplificada de un panorama mucho más complejo. Sin embargo, aunque esta falencia puede leerse como algo negativo, es a la vez una excelente oportunidad para estimular la investigación y los muestreos a nivel nacional, ya que es muy probable que en el $89.38 \%$ del país que aun no se ha muestreado, puedan reportarse ampliaciones de distribución y descubrirse nuevas especies.

Adicionalmente, a pesar del incremento de trabajos y publicaciones y de la existencia de varios protocolos que aclaran y especifican las metodologías adecuadas de muestreo (Villareal et al., 2004; Larsen \& Forsyth, 2005; Noriega \& Fagua, 2009; Cultid et al., 2012), siguen realizándose estudios que no cumplen con los requerimientos de un muestreo estándar al no tener en cuenta aspectos básicos como el 
número mínimo de trampas o la distancia mínima entre ellas, lo cual evidencia la necesidad de establecer una metodología unificada a nivel nacional. Una de las más importantes conclusiones de este análisis, es que es necesario hacer una transición radical de muestreos rápidos hacia protocolos exhaustivos, completos y estandarizables que permitan comparar los resultados entre diferentes estudios y así poder llegar a conocer a fondo la diversidad existente de este grupo en el territorio colombiano.

De igual manera, sería interesante poder articular este tipo de análisis con otros grupos faunísticos en el país, como hormigas (Hymenoptera: Formicidae), mariposas diurnas (Lepidoptera: Rhopalocera), libélulas (Odonata) o escarabajos fitófagos (Cetoniinae, Dynastinae, Melolonthinae y Rutelinae) y de esta manera poder comparar patrones de muestreo y consolidar áreas prioritarias de estudio a nivel nacional. Finalmente, esperamos que este análisis estimule el estudio y uso de este grupo de insectos en trabajos de evaluación de impacto ambiental, consolidando su utilización como bioindicadores del grado de conservación y funcionalidad de un ecosistema.

\section{AGRADECIMIENTOS}

A los directores, curadores y asistentes de curaduría de las colecciones revisadas: Claudio Fernández, Alfonso Villalobos, Carlos Sarmiento, Guillermo Castillo, Andrés Ramiro Hidalgo, Rodrigo Torres, Giovanny Fagua, Nancy Carrejo, Patricia Chacón, Marta Wolff, Sandra Pérez, Juliana Cardona, Fredy Molano, Roberto Quiñones, Fernando Valencia, Daniel Miranda, Emilio Realpe, Jhon Jairo Idarraga, Jhon Albéiro Quiroz, José Maria Riascos, German Parra, Miguel Antonio Gualteros, Édison Torrado, Jhon Cesar Neita, Gilberto Morales Soto, Roberto León Ojalvo, Luis Carlos Pardo, Camilo Andrade, Anderson Muñoz, Sandra Cabezas, Cesar Cardona, Magnolia del Pilar Cano, Oscar Efraín Ortega, Martha Londoño, Celsa García, Hermano Roque Casallas y Fernando Vallejo. A Giovanny Fagua, Tomas
Walsburger, Julio Mario Hoyos, Angela Amarillo, Daniel Monroy y Andres Etter por sus importantes comentarios y aportes a este trabajo. A Patricia Pineda y Daniel Monroy por su ayuda en la elaboración de los mapas y en el cálculo del porcentaje de cobertura. A Emiro Martínez, Alejandra Bedoya, Jorge Eliecer Olarte, Rodrigo Sarmiento, Paola Delgado, Walter David Guzman, Asdrubal Redondo, Andrea Velandia, Greisha Salcedo y Silvia Ardila por toda la información y ayuda suministrada. Especialmente a Alejandro Lopera, Bedir Martínez y Carolina Giraldo por sus valiosos aportes, correcciones y constantes revisiones al manuscrito que mejoraron significativamente su calidad. A David Morris por la revisión del abstract. A Gerardo Lamas quien propició la idea de este artículo.

\section{RESUMEN}

Una de las principales limitantes para generar estrategias para la conservación, es la falta de información y la dificultad para identificar vacíos de conocimiento, que faciliten el entendimiento de un grupo taxonómico. En este trabajo desarrollamos el diagnóstico sobre el grado de cobertura del muestreo de escarabajos coprófagos en Colombia. Se recopiló la bibliografía, utilizando 12 bases de datos y se revisaron 16940 especímenes depositados en 26 colecciones. Se encontraron 232 especies, en 386 localidades, con un porcentaje de cobertura del $10.62 \%$. Se evidencia en los últimos años un incremento en el número de estudios, sin embargo solo 64 localidades tienen publicaciones. Se encontraron registros para todos los departamentos, siendo los mejor muestreados: Cundinamarca, Antioquia, Valle del Cauca y Boyacá. Los ecosistemas con mayor número de publicaciones son el bosque húmedo premontano Andino, el bosque húmedo montano Andino y el bosque húmedo del Pacífico. Sin embargo, existen muchos ecosistemas con muy pocos estudios como los manglares, zonas desérticas, savanas naturales, morichales, páramos, bosques inundables y sistemas agrícolas. La región biogeográfica con mayor número de localidades es la Andina, seguida del Chocó-Magdalenense y la Amazonía. A nivel general, persiste un alto nivel de submuestreo. Se evidencia la necesidad de ampliar la cobertura del muestreo a nivel nacional, enfocando los esfuerzos en aquellas áreas submuestreadas con importancia a nivel de conservación.

Palabras clave: colecciones, distribución geográfica, localidades, muestreo, regiones biogeográficas, Scarabaeidae. 


\section{REFERENCIAS}

Acevedo, A. A., \& Aguas, A. J. (2007). Distribución y abundancia de escarabajos coprófagos en tres comunidades de selva alto andina, municipio de Pamplona, Norte de Santander, Colombia. Trabajo de pregrado. Universidad de Pamplona, Pamplona, Colombia.

Agudelo, L. F. (2012). Inventario preliminar de escarabajos coprófagos (COLEOPTERA: SCARABAEINAE) asociados a una pastura de Micay (Axonopus micay) y al Parque Natural Cerro Gobia en Quinchía Risaralda. Trabajo de pregrado. Programa de Zootecnia, Corporación Universitaria Santa Rosa de Cabal, Pereira, Colombia.

Alvarado, F. A. (2008). Variación altitudinal de la composición y riqueza de escarabajos copro - necrófagos (Insecta: Coleoptera: Scarabaeidae: Scarabaeinae) en un transecto vertical en un bosque montano de la vertiente oriental del Parque Nacional Natural El Cocuy, vertiente oriental (Boyacá, Arauca - Colombia). Trabajo de pregrado. Universidad Pedagógica y Tecnológica de Colombia, Tunja, Colombia.

Álvarez, M., Escobar, F., Mendoza, H., \& Villarreal, H. (1999). Caracterización de la biodiversidad en áreas prioritarias de la vertiente oriental de la Cordillera Oriental. (Informe Técnico). Villa de Leyva, Colombia: Grupo GEMA, Programa de Inventarios de Biodiversidad, Instituto Alexander von Humboldt.

Amat, G. G., \& Trujillo, D. (2004). Escarabajos (Coleoptera: Scarabaeoidea) en el Chocó Biogeográfico. In J. O. Rangel (Ed.), Colombia Diversidad Biótica IV. El Chocó biogeográfico/Costa Pacífica (pp. 745754). Universidad Nacional de Colombia, Bogotá, Colombia.

Amat, G. G., Lopera, A. T., \& Amézquita, S. (1997). Patrones de distribución de escarabajos coprófagos (Coleoptera: Scarabaeidae) en relicto del bosque alto andino Cordillera Oriental de Colombia. Caldasia, 19(1-2), 191-204.

Amell, Y. C. (2012). Esctructura del ensamblaje de escarabajos coprófagos (Coleoptera: Scarabaeinae) en las cinco subregiones del departamento de Sucre, Colombia. Trabajo de pregrado. Universidad del Magdalena, Santa Marta, Colombia.

Amézquita, M. S., Forsyth, A., Lopera, A., \& Camacho, A. (1999). Comparación de la composición y riqueza de especies de escarabajos coprófagos (Coleoptera: Scarabaeidae) en remanentes de bosque de la Orinoquía Colombiana. Acta Zoológica Mexicana (n.s.), $76,113-126$.

Amorim, D. S. (2001). Dos Amazonías. In J. B. Llorente \& J. J. Morrone (Eds.), Introducción a la biogeografia en Latinoamérica: Teorías, conceptos y aplicaciones (pp. 245-255). México: Facultad de Ciencias, UNAM, Mexico, D.F.
Arango, L., Montes, J. M., López, D. A., \& López, J. O. (2007). Mariposas (Lepidoptera: Papilionoidea, Hesperoidea), escarabajos coprófagos (Coleoptera: Scarabaeinae) y hormigas (Hymenoptera: Formicidae) del Ecoparque Alcázares - Arenillo (Manizales, Caldas - Colombia). Boletín Científico Centro de Museos Museo de Historia Natural, 11, 390-409.

Arango, L., \& Montes, J. M. (2009). Caracterización entomológica parcial de la cuenca del río la Miel en el departamento de Caldas (Colombia). Boletín Científico Centro de Museos Museo de Historia Natural, 13(2), 249-268.

Arbeláez-Cortés, E. (2013). Knowledge of Colombian biodiversity: published and indexed. Biodiversity and Conservation, 22, 2875-2906.

Ardila, J. M. (2005). Diversidad de escarabajos coprófagos (Coleoptera: Scarabaeinae) en transectos borde - interior en un bosque montano (Medina, Cundinamarca). Trabajo de pregrado. Pontificia Universidad Javeriana, Bogotá, Colombia.

Ardila, S. (2011). Diversidad y distribución de escarabajos coprófagos (Coleoptera: Scarabaeidae) en un gradiente altitudinal en la Granja Experimental Villa Marina, Norte de Santander. Trabajo de pregrado. Universidad de Pamplona, Pamplona, Colombia.

Arias-Buriticá, J. A., \& Vaz-De-Mello, F. (2013). Dichotomius ribeiroi (Pereira, 1954) (Coleoptera: Scarabaeidae: Scarabaeinae): redescripción y anotaciones taxonómicas de la especie. Caldasia, 35(1), 209-217.

Arias-Buriticá, J. A., Delgado-Gómez, P., González, F. A., \& Vaz-De-Mello, F. Z. (2011). Nuevos registros de escarabajos coprófagos (Coleoptera: Scarabaeidae: Scarabaeinae) para el departamento de Chocó (Colombia). Acta Zoológica Mexicana (n.s.), 27(3), 875-878.

Arias, J. A. (2008). Estructura de la comunidad de escarabajos coprófagos (Scarabaeidae: Scarabaeinae) en tres paisajes fragmentados en Arcabuco (Boyaca). Trabajo de pregrado. Universidad Pedagógica y Tecnológica de Colombia, Tunja, Colombia.

Arias, J. A. (2011). Revisión taxonómica de la sección "buqueti", Dichotomius, Hope, 1838 (Coleoptera: Scarabaeidae: Scarabaeinae). Tesis de Maestría. Universidad Nacional de Colombia, Bogotá, Colombia.

Arias, J. A. \& Medina, C. A. (2014). Tres nuevas especies de Cryptocanthon Balthasar, 1942 (Coleoptera: Scarabaeidae: Scarabaeinae) para Colombia. Caldasia, 36(1), 165-180.

Arrow, G. (1933). The genus Uroxys (Coleoptera, Copridae), with descriptions of some new species. Annals and Magazine of Natural History, 10(11), 385-399.

Barón, L. H. (2008). Plan de mercadeo para la comercializacion de abono orgánico de escarabajo, en la zona centro de Boyacá, para aporte de la empresa "Tierra 
Viva". Trabajo de pregrado. Universidad Pedagógica y Tecnológica de Colombia, Tunja, Colombia.

Barraza, J., \& Montes, J. (2009). Ensamblaje de escarabajos coprófagos en un paisaje de Bosque Seco Tropical en Bahía Concha, Santa Marta, Colombia. Trabajo de pregrado. Universidad del Atlántico, Barranquilla.

Barraza, J., Montes, J., Martínez, N., \& Deloya, C. (2010). Ensamblaje de escarabajos coprófagos (Scarabaeidae: Scarabaeinae) del Bosque Tropical Seco, Bahía Concha, Santa Marta (Colombia). Revista Colombiana de Entomología, 36(2), 285-291.

Bedoya, A. (2008). Efecto de la complejidad del hábitat en la composición de la comunidad de escarabajos coprófagos (Scarabaeidae: Scarabaeinae) en bosques premontanos del área de influencia de la central hidroeléctrica "porce II". Trabajo de pregrado. Facultad de Ciencias Exactas y Naturales, Universidad de Antioquia, Medellin, Colombia.

Bernal, M. C. (2005). Primera lista comentada y conformación de una base de datos de los escarabajos (Coleoptera: Scarabaeoidea) de Boyacá, Colombia. Trabajo de pregrado. Universidad Pedagógica y Tecnológica de Colombia, Tunja, Colombia.

Blanco, A., Navarro, K., Solis, C., Gutierrez, L. C., \& Bonilla, M. A. (2013). Anuros del bosque seco tropical (Caribe Colombiano) ingieren al escarabajo exótico Digitonthophagus gazella (Scarabaeinae: Onthophagini). Entomotropica, 28(3), 227-232.

Bohórquez, J. C. (2003). Contribución al conocimiento de la comunidad de coleópteros coprófagos (Coleoptera: Scarabaeidae) en la reserva forestal de Colosó (Sucre). Trabajo de pregrado. Facultad de Ciencias Básicas, Universidad del Atlántico, Barranquilla, Colombia.

Bohórquez, J. C., \& Montoya, J. (2009). Abundancia y preferencia trófica de Dichotomius belus (Coleoptera: Scarabaeidae) en la reserva forestal de Colosó, Sucre. Boletín del Museo de Entomología de la Universidad del Valle, 10(1), 1-7.

Botina, C. P., \& Castillo, J. I. (2012). Estructura de las comunidades de escarabajos coprófagos (Coleoptera: Scarabaeidae: Scarabaeinae) en un gradiente altitudinal en el departamento de Nariño al suroccidente de Colombia. Trabajo de pregrado. Universidad de Nariño, Pasto, Colombia.

Bouchard, P., Bousqueti, Y., Davies, A. E., Alonso-Zarazaga, M. A., Lawrence, J. F., Lyal, C. H., Newton, A. F., Reid, C., Schmitt, M., Ślipiński, S., \& Smith, A. B. (2011). Family-group names in Coleoptera (Insecta). ZooKeys, 88, 1-972.

Bustos, L. F. (2001). Preferencias alimenticias de los escarabajos coprófagos (Scarabaeinae) en un remanente de bosque seco tropical al norte del Tolima. Trabajo de pregrado, Universidad de Los Andes, Bogotá, Colombia.
Bustos-Gómez, L. F., \& Lopera, A. (2003). Preferencia por cebo de los escarabajos coprófagos (Coleoptera: Scarabaeidae: Scarabaeinae) de un remanente de bosque seco tropical al norte del Tolima (Colombia). In G. Onore, P. Reyes, \& M. Zunino (Comp.), Escarabaeidos de Latinoamérica: Estado del conocimiento (pp. 59-56). m3m-Monografías Tercer Milenio, 3. Sociedad Entomológica Aragonesa. Zaragoza, España.

Camacho, R. A. (1999). Usos de las cercas vivas por parte de los escarabajos coprófagos (Escarabaeidae: Scarabaeinae) en un ambiente, fragmentado del piedemonte llanero, Meta, Colombia. Trabajo de pregrado. Pontificia Universidad Javeriana, Bogotá, Colombia.

Cambefort, Y. (1991a). Biogeography and evolution. In I. Hanski \& Y. Cambefort (Eds.), Dung Beetle Ecology (pp. 51-68). New Jersey: Princeton University Press.

Cambefort, Y. (1991b). From saprophagy to coprophagy. In I. Hanski \& Y. Cambefort (Eds.), Dung Beetle Ecology (pp. 21-35). New Jersey: Princeton University Press.

Camero, E. (2010). Los escarabajos del género Eurysternus Dalman, 1824 (Coleoptera: Scarabaeidae) de Colombia. Boletín de la Sociedad Entomológica Aragonesa (S.E.A.), 46, 147-179.

Camero, E., \& Lobo, J. M. (2010). Distribución conocida y potencial de las especies del género Eurysternus Dalman, 1824 (Coleoptera: Scarabaeidae) de Colombia. Boletin de la Sociedad Entomológica Aragonesa (S.E.A.), 47, 257-264.

Cupello, M., \& Vaz-De-Mello, F. Z. (2013). Taxonomic revisión of the South American dung beetle genus Gromphas Brullé, 1837 (Coleoptera: Scarabaeidae: Scarabaeinae: Phanaeini: Gromphadina). Zootaxa, 3722(4), 439-482.

Cardenas, J. S. (2011). Escarabajos coprófagos (Scarabaeidae: Scarabaeinae) en dos fragmentos de bosque y su matriz circundante en el caribe colombiano. Trabajo de pregrado. Universidad Pedagógica y Tecnológica de Colombia, Tunja, Colombia.

Cárdenas-Bautista, J., Morales-Castaño, I. T., \& CarvajalCogollo, J. E. (2012). Escarabajos coprófagos (Scarabaeidae: Scarabaeinae) en dos fragmentos de bosque y su matriz circundante en el caribe colombiano. In J. O. Rangel-Ch (Ed.), Colombia Diversidad Biótica XII: La región Caribe de Colombia (pp. 821-831). Instituto de Ciencias Naturales, Bogotá, Colombia.

Casas, A. C., \& Pineda, N. T. (2008). Estudio de la actividad diaria de un ensamblaje de escarabajos coprófagos (Coleoptera: Scarabaeidae) en un bosque altoandino. Trabajo de pregrado. Universidad Militar Nueva Granada, Bogotá, Colombia.

Castaño, M. (2003). Comparación de los escarabajos coprófagos (Scarabaeidae: Scarabaeinae) en plantaciones de pino y bosques nativos, San Antonio de 
Prado, Medellín, Antioquia. Trabajo de pregrado. Universidad de Antioquia, Medellín, Colombia.

Castellanos, M. C., Escobar, F., \& Stevenson, P. R. (1999). Dung beetles (Scarabaeidae: Scarabaeinae) attracted to woolly monkey (Lagothrix lagothricha Humboldt) dung at Tinigua National Park, Colombia. The Coleopterists Bulletin, 53(2), 155-159.

Castellanos, M. L., Pardo-Locarno, L. C., Carabalí, A., \& Doria, C. (2011). Algunas características de macrofauna del suelo en la Serranía de la Macuira, Guajira, Colombia. Revista Agricultura Tropical, 34(3,4), 98-106.

Castillo, R., \& Osorio, D. (2006). Diversidad y composición de dípteros, coleópteros e himenópteros asociados a excremento humano en el Parque Nacional Natural El Tuparro (Vichada - Colombia). Trabajo de pregrado. Universidad Tecnológica de Colombia y Instituto de Investigación de Recursos Biológicos Alexander von Humboldt.

Castillo, R. M., Lara, M. I., \& Olivares, J. C. (2011). Diversidad preliminar de escarabajos coprófagos (Coleoptera: Scarabaeinae), atráidos por excremento humano en el Calvario, Meta. Conexión Agropecuaria JDC, 1(1), 17-33.

Castillo, S. (2013). Efecto de la perturbación en la tasa de remoción del ensamblaje de escarabajos coprófagos (Coleoptera: Scarabaeinae) en un Bosque Seco Tropical, Reserva Natural Kalashe-Kalabia, Santa Marta, Colombia. Trabajo de pregrado. Universidad del Magdalena, Santa Marta, Colombia.

Castro, D. J. (2001). Estudio del estado de la comunidad de escarabajos coprófagos (Coleoptera: Scarabaeidae: Scarabaeinae y Coprinae) en áreas de colonización de la selva amazónica, departamento del Guaviare, Colombia. Trabajo de pregrado. Universidad Nacional de Colombia, Bogotá.

Concha-Lozada, C., Gallego, M., \& Pardo-Locarno, L. C. (2010). Fragmentación de ecosistemas montanos e impactos estructurales y poblacionales sobre la comunidad de escarabajos coprófagos (Coleoptera: Scarabaeidae) en el alto Río Cauca, Popayán, Colombia. Boletín Científico Centro de Museos de Historia Natural, 14(1), 43-55.

Cracraft, J., \& Prum R. O. (1988). Patterns and processes of diversification: speciation and historical congruence in some Neotropical birds. Evolution, 42, 603-620.

Crisci, J. V., Posadas, P., Katinas L., \& Miranda, D. R. (1999). Estrategias evolutivas para la conservación de la biodiversidad en América del Sur Austral. In S. D. Matteucci, O. T. Soebrig, J. Morello, \& G. Halffter (Eds.). Biodiversidad y uso de la tierra. Conceptos y ejemplos de Latinoamérica (pp. 175-198). Buenos Aires, Argentina: Eudeba.

Cultid, C. A. (2007). Ritmos de actividad diaria de vuelo en una comunidad de coleópteros coprófagos
(Scarabaeidae: Scarabaeinae) de un paisaje andino, Risaralda, Colombia. Trabajo de pregrado. Universidad del Valle, Cali, Colombia.

Cultid, C. A., Medina, C. A., Martínez, B. G., Escobar, A. F., Constantino, L. M., \& Betancur, N. J. (2012). Escarabajos coprófagos (Scarabaeinae) del eje cafetero: Guía para el estudio etológico. (Ed.) WCS, CENICAFE \& Federación Nacional de Cafeteros de Colombia. Villa Maria, Caldas, Colombia.

Cultid, C. A., \& Giraldo, A. (2012). Coprophanaeus morenoi Arnaud, 1982 (Coleoptera: Scarabaeidae: Scarabaeinae) in the National Natural Park (Colombian Pacific Ocean). Boletín Cientifico Museo Historia Natural Universidad de Caldas, 16(1), 261-265.

Delgado, C. M. (1998). Estudio de las comunidades de insectos con énfasis en los escarabajos copronecrófagos (Coleoptera: Scarabaeidae) en cuatro estados sucesionales en la zona de influencia al proyecto hidroeléctrico Porce II. Trabajo de pregrado. Universidad Nacional de Colombia, Medellín, Colombia.

Delgado-Gómez, P. (2012). Influencia del hábitat sobre el ensamblaje de escarabajos coprófagos (Scarabaeidae: Scarabaeinae) en bosques secos en el municipio de Chimichagua (Cesar). Tesis de Maestría. Universidad Nacional de Colombia, Bogotá, Colombia.

Delgado-Gómez, P., Lopera, A., \& Rangel-Ch., J. O. (2012). Variación espacial del ensamblaje de escarabajos coprófagos (Scarabaeidae: Scarabaeinae) en remanentes de bosque seco en Chimichagua (Cesar, Colombia). In J.O. Rangel-Ch. (Ed.), Colombia Diversidad Biótica XII: La región Caribe de Colombia (pp. 833-849). Instituto de Ciencias Naturales, Bogotá, Colombia.

Escobar, A. (2009). Taxonomía y variación morfológica del complejo de especies del género Uroxys (Scarabaeidae: Scarabaeinae) en el sistema regional de áreas protegidas del eje cafetero (SIRAP-EP), Colombia. Trabajo de pregrado. Universidad del Valle, Cali, Colombia.

Escobar, F. (1994). Excremento, coprófagos y deforestación en bosques de montaña al suroccidente de Colombia. Trabajo de pregrado. Universidad del Valle, Cali, Colombia.

Escobar, F. (1997). Estudio de la comunidad de coleópteros coprófagos (Scarabaeidae) en un remanente de bosque seco al norte del Tolima, Colombia. Caldasia, 19(3), 419-430.

Escobar, F. (2000a). Diversidad de coleópteros coprófagos (Scarabaeidae: Scarabaeinae) en un mosaico de hábitats en la Reserva Natural Nukak, Guaviare, Colombia. Acta Zoológica Mexicana (n.s.), 79, 103-121.

Escobar, F. (2000b). Diversidad y distribución de los escarabajos del estiércol (Coleoptera: Scarabaeidae: Scarabaeinae) de Colombia. In F. M. Piera, J. J. Morrone, \& A. Melic (Eds.), Hacia un proyecto Cyted para el 
inventario y estimación de la diversidad entomológica en Iberoamerica: PrIBES-2000 (pp. 197-210). Monografías Tercer Milenio I, SEA, Zaragoza.

Escobar, F. (2003a). Feeding habits and distributional records of 11 species of Neotropical Scarabaeinae (Coleoptera: Scarabaeidae). The Coleopterist Bulletin, 57(2), 131-132.

Escobar, F. (2003b). Selección de hábitat y comportamiento sexual de Sulcophanaeus velutinus (Murray 1856) (Coleoptera: Scarabaeidae: Scarabaeinae) en un bosque de montaña en Colombia. Acta Zoológica Mexicana (n.s.), 90, 307-310.

Escobar, F. (2004). Diversity and composition of dung beetle (Scarabaeinae) assemblages in a heterogeneous Andean landscape. Tropical Zoology, 17, 123-136.

Escobar, F., \& Chacón De Ulloa, P. (2000). Distribución espacial y temporal en un gradiente de sucesión de la fauna de coleópteros coprófagos (Scarabaeidae, Aphodiinae) en un bosque tropical montano, Nariño - Colombia. Revista de Biología Tropical, 48(4), 961-975.

Escobar, F., \& Medina, C. (1996). Coleópteros coprófagos (Scarabaeidae) de Colombia: estado actual de su conocimiento. In G. Amat, G. Andrade, \& F. Fernández (Eds), Insectos de Colombia. Volumen I (pp. 93-116). Academia Colombiana de Ciencias Exactas, Físicas y Naturales. Colección Jorge Alvarez Lleras. Bogotá, Colombia: Editora Guadalupe.

Escobar, F., Lobo, J. M., \& Halffter, G. (2005). Altitudinal variation of dung beetle (Scarabaeidae: Scarabaeinae) assemblages in the Colombian Andes. Global Ecology and Biogeography, 14, 327-337.

Escobar, F., Lobo, J. M., \& Halffter, G. (2006). Assessing the origin of neotropical mountain dung beetle assemblages (Scarabaeidae: Scarabaeinae): the comparative influence of vertical and horizontal colonization. Journal of Biogeography, 33, 1793-1803.

Escobar, F., Halffter, G., \& Arellano, L. (2007). From forest to pasture: an evaluation of the influence of environment and biogeography on the structure of dung beetle (Scarabaeinae) assemblages along three actitudinal gradients in the Neotropical region. Ecography, 30, 193-208.

Esparza, A. (2006). Composición y riqueza de escarabajos coprófagos (Coleoptera: Scarabaeidae: Scarabaeinae) en un gradiente altitudinal de selva húmeda tropical (P.N.N. Catatumbo/Bari). Trabajo de pregrado. Universidad Industrial de Santander, Bucaramanga, Colombia.

Esparza, A., \& Amat, G. (2007). Composición y riqueza de escarabajos coprófagos (Coleoptera: Scarabaeidae) en un gradiente actitudinal de selva húmeda tropical del Parque Nacional Natural Catatumbo-Barí (Norte de Santander, Colombia). Actualidades Biológicas, 29(87), 181-192.
Espitia, D. P. (2010). Diversidad de escarabajos coprófagos y hormigas en sistemas ganaderos: comparación entre sistema tradicional y silvopastoril en los municipios de Belén y Paipa (Pantano de Vargas) - Boyacá. Trabajo de pregrado. Universidad Pedagógica y Tecnológica de Colombia, Tunja, Colombia.

Etter, A. A. (1993). Diversidad ecosistémica en Colombia hoy. Nuestra Diversidad Biológica. Cerec, 1, 47-66.

Etter, A. A., \& Wyngaarden, W. V. (2000). Patterns of landscape transformation in Colombia, with emphasis in the Andean Region. Suecia Ambio, 29(7), 443-450.

Etter A. A., Mcalpine, C., \& Possingham, H. P. (2008). A historical analysis of the spatial and temporal drivers of landscape change in Colombia since 1500. Annals of the Association of American Geographers, 98(1), $2-23$.

Etter, A., Mcalpine, C., Wilson, K., Phinn, S., \& Possingham, H. (2006). Regional patterns of agricultural land use and deforestation in Colombia. Agriculture, Ecosystems \& Environment, 114(2-4), 369-386.

Fernández, F. (2011). The greatest impediment to the study of biodiversity in Colombia. Caldasia, 33(2), 1-3.

Fuentes, P. V. (2004). Composición y distribución espaciotemporal de escarabajos coprófagos (Coleoptera: Scarabaeidae: Scarabaeinae) en el bosque municipal de Mariquita-Tolima. Trabajo de pregrado. Universidad Nacional de Colombia, Bogotá, Colombia.

Fuentes, P. V., \& Camero, E. (2006). Estudio de la fauna de escarabajos coprófagos (Coleoptera: Scarabaeidae) en un bosque húmedo tropical de Colombia. Entomotropica, 21(3), 133-143.

Gardner, T. A., Hernández, M. I. M., Barlow, J., \& Peres, C. A. (2008). Understanding the biodiversity consequences of hábitat change: the value of secondary and plantation forests for neotropical dung beetles. Journal of Applied Ecology, 45, 883-893.

García, H. G., \& Ospino, D. A. (2005). Diversidad de escarabajos coprófagos (Coleoptera: Scarabaeinae) en un gradiente altitudinal en la vertiente Noroccidental de la Sierra Nevada de Santa Marta, Colombia. Trabajo de pregrado. Universidad del Magdalena, Santa Marta, Colombia.

García, J. C., \& Pardo-Locarno, L. C. (2004). Escarabajos Scarabaeinae saprófagos (Coleoptera: Scarabaeidae) en un bosque muy húmedo premontano de los Andes Occidentales Colombianos. Ecología Aplicada, 3(1, 2), 59-63.

Garzón, A. M., Galeano, J., \& Vélez, S. (2003). Composición y diversidad de la comunidad de escarabajos coprófagos (Scarabaeidae: Scarabaeinae) en un paisaje agropecuario del municipio de Filandia, Quindío. Trabajo de pregrado. Licenciatura en Biología y Educación Ambiental. Universidad del Quindío, Armenia, Colombia. 
Garzón-Orduña, I. J., \& Miranda-Esquivel, D. R. (2007). Amazonian relationships: an example of a complex area and a complex problem. Biogeografía, 1, 17-20.

Gasca, H. J. (2005). El significado de los escarabajos (Coleoptera: Scarabaeoidea) en una comunidad Uitoto de Leticia, Amazonas (Colombia): una exploración preliminar a su conocimiento etnoentomológico. Boletín Sociedad Entomológica Aragonesa, 36, 309-315.

Gasca, H. J., \& Ospina, M. F. (2000). Estudio preliminar de la composición de la comunidad de escarabajos coprófagos (Coleoptera: Scarabaeidae) de un bosque altoandino de Albán (Cundinamarca, Colombia). Acta Biológica Colombiana, 5(2), 19-22.

GEMA (Grupo de Exploración y Monitoreo Ambiental). (2002). Caracterización biológica del sector de Sisavita, Municipio de Cucutilla, Norte de Santander. Instituto de Investigación de Recursos Biológicos Alexander von Humboldt (IAVH), Villa de Leyva, Colombia.

Gill, B. D. (1991). Dung beetles in tropical American forests. In I. Hanski, \& Y. Cambefort (Eds.), Dung Beetle Ecology (pp. 211-230). New Jersey, USA: Princeton University Press, Princeton.

Giraldo, C. (2007). Escarabajos estercoleros amigos de la ganadería. Carta de Fedegán, 100, 74-76.

Giraldo, C., Londoño, J. F., \& Buitrago, J. A. (2005). Patrones espaciales de diversidad de escarabajos coprófagos (Scarabaeidae: Scarabaeinae) en trece reservas de la sociedad civil del departamento del Quindío. Trabajo de pregrado. Licenciatura en Biología y Educación Ambiental. Universidad del Quindío, Armenia, Colombia.

Giraldo, C., \& Murgueitio, E. (2010). Escarabajos estercoleros: los cinceles y rastrillos de las tierras ganaderas. Carta de Fedegán, 116, 76-78.

Giraldo, C., Escobar, F., Chará, J. D., \& Calle, Z. (2011). The adoption of silvopastoral systems promotes the recovery of ecological processes regulated by dung beetles in the Colombian Andes. Insect Conservation and Diversity, 4(2), 115-122.

Girón-Vanderhuck, M., Molina-Rico, J., \& Aguirre-Obando, O. A. (2010). Cambios en las propiedades químicas del suelo con la utilización de Dichotomius satanas (Harold, 1867) (Coleoptera: Scarabaeidae: Scarabaeinae) en condiciones de invernadero. Revista de Investigaciones Universidad del Quindio, 21, 43-54.

González, J. A., \& Copete, F. G. (2012). Diversidad de escarabajos copro-necrófagos (Scarabaeidae: Scarabaeinae) presente en la finca cafetera el paraíso del municipio de la Celia, Risaralda. Trabajo de pregrado. Programa de Zootecnia, Corporación Universitaria Santa Rosa de Cabal, Pereira, Colombia.
González, J. J., Etter, A. A., Sarmiento, A., \& Cabrera, E. (2011). Análisis de tendencias y patrones espaciales de deforestación en Colombia. Instituto de Estudios Ambientales - Ideam.

González, F. A. (2008). Los subgéneros Calhyboma Kolbe, 1893, Hybomidium Shipp 1897 y Telhyboma Kolbe 1893 (Deltochilum: Scarabaeidae) en Colombia. Trabajo de pregrado. Universidad Pedagógica y Tecnológica de Colombia, Tunja, Colombia.

González, F. A., Molano, F., \& Medina, C. A. (2009). Los subgéneros Calhyboma, Hybomidium y Telhyboma (Coleoptera: Scarabaeidae: Sacrabaeinae: Deltochilum) en Colombia. Revista Colombiana de Entomología, 35(2), 253-274.

Halffter, G. (1991). Historical and ecological factors determining the geographical distribution of beetles (Coleoptera: Scarabaeidae: Scarabaeinae). Folia Entomológica Mexicana, 82, 195-238.

Halffter, G., \& Favila, M. E. (1993). The Scarabaeidae (Insecta: Coleoptera) an animal group for analysing, inventoryng and monitoring biodiversity in tropical rainforest and modified landscapes. Biology International, 27, 1-21.

Halffter, G., \& Matthews, E. G. (1966). The natural history of dung beetles of the subfamily Scarabaeinae (Coleoptera: Scarabaeidae). Folia Entomológica Mexicana, 12-14, 1-312.

Hernández, E. (2011). Dinamica espacio-temporal del ensamblaje de escarabajos coprófagos (Scarabaeidae: Scarabaeinae) en el relleno sanitario clausurado de Veracruz (RSCV) Santa Marta - Colombia. Trabajo de pregrado. Universidad del Magadalena, Santa Marta, Colombia.

Hernández, J. C., Hurtado, A. G., Quijano, R. Q., \& Walschburger, T. B. (1992). Unidades biogeográficas de Colombia. In G. Halffter (Ed.), La Diversidad Biológica de Iberoamerica (pp. 41-43). Acta Zoológica Mexicana, vol. Especial, México.

Hernández, A., \& Elejalde, J. A. (2000). Diversidad de escarabajos coprófagos (Scarabaeidae: Sacrabaeinae) en sistemas cafeteros. Trabajo de pregrado. Licenciatura en Biología y Educación Ambiental. Universidad del Quindío, Armenia, Colombia.

Herrera, E. R., \& Gutiérrez, J. M. (2005). Escarabajos necrófagos (Col, Scarabaeidae) como posible bioindicadores del estado sucesional en el bosque seco tropical del Jardín Botánico, corregimiento Mateguadua, Tuluá-Valle. Trabajo de pregrado. Universidad Central del Valle, Tuluá, Colombia.

Herrera, G. A. (2005). Algunos aspectos ecológicos de la subfamilia Scarabaeinae (Coleoptera: Scarabaeidae) en un remanente de Bosque Seco Tropical (Bs-T) del cerro La Vieja, municipio de Piojó, departamento del Atlántico, Colombia. Trabajo de pregrado. Universidad del Atlántico, Barranquilla, Colombia. 
Higuera-Díaz, M., \& Ospina-Correa, M. (2009). Insectos. In H. Villarreal-Leal, M. Álvarez-Rebolledo, M. Higuera-Díaz, J. Aldana-Domínguez, J. D. BogotáGregory, F. A. Villa-Navarro, P. von Hildebrandt, A. Prieto-Cruz, J. A. Maldonado-Ocampo, A. M. Umaña-Villaveces, S. Sierra, \& F. Forero (Eds.). Caracterización de la biodiversidad de la selva de Matavén (sector centro-oriental) Vichada, Colombia (pp. 115139). Bogotá, Colombia: Instituto de Investigación de Recursos Biológicos Alexander von Humboldt y Asociación de Cabildos y Autoridades Tradicionales Indígenas de la selva de Matavén (Acatisema).

Howden, H. F., \& Campbell, J. M. (1974). Observations on some Scarabaeoidea in the Colombian Sierra Nevada de Santa Marta. The Coleopterists Bulletin, 28(3), 109-114.

Howden, H. F., \& Nealis, V. G. (1975). Effects of clearing in a tropical rain forest on the composition of the coprophagous scarab fauna (Coleoptera). Biotropica, $7(2), 77-83$.

Huertas, B., Arias, J., \& Pardo-Locarno, L. C. (2003). Estudio preliminar de los escarabajos coprófagos (Coleoptera: Scarabaeidae) de la Serranía de Los Churumbelos, Cauca (Expedición Colombia 1998). Boletín Científico Museo Historia Natural Universidad de Caldas, 7, 215-218.

IGAC (Instituto Geográfico Agustín Codazzi) (2013). Cartografía básica de Colombia 1:100.000. Recuperado de http://geocarto.igac.gov.co/geoservicios/cien_mil/ wms.

Jiménez, L. A., \& Mendieta, W. J. (2005). Coleopterofauna de las formaciones subxerofíticas tropicales de los cerros de Santa Marta-Colombia: registro fotográfico. Trabajo de pregrado. Universidad del Magdalena, Santa Marta, Colombia.

Jiménez, L., Mendieta, W., García, H., \& Amat, G. (2008). Notas sobre los escarabajos coprófagos (Coleoptera: Scarabaeinae) en ambientes secos de la región de Santa Marta, Colombia. Acta Biológica Colombiana, 13(2), 203-208.

Larsen, T. H., \& Forsyth, A. (2005). Trap spacing and transect design for dung beetle biodiversity studies. Biotropica, 37, 322-325.

Laverde, L. J., Castellanos, M. C., \& Stevenson, P. (2002). Dispersión secundaria de semillas por escarabajos coprófagos (Scarabaeidae) a partir de heces de churucos (Lagothrix lagothricha) en el Parque Nacional Tinigua, Colombia. Universitas Scientiarum, 1(1), 17-24.

Lopera, A. (1996). Distribución y diversidad de escarabajos coprófagos (Scarabaeidae) en tres relictos de bosque altoandino (Cordillera Oriental, Vertiente Occidental), Colombia. Trabajo de pregrado. Pontificia Universidad Javeriana, Bogotá, Colombia.
López, D. P. (2010). Estado de recuperación de las comunidades de escarabajos coprófagos (Coleoptera: Scarabaeidae) en remanentes de bosque en la Orinoquía colombiana. Trabajo de pregrado. Universidad Pedagógica y Tecnológica de Colombia, Tunja, Colombia.

López, R., \& Martínez, E. (2011). Ensamblaje de Escarabajos coprófagos (Coleoptera: Scarabaeidae: Scarabaeinae) en cultivos de banano bajo manejo orgánico en Buritaca, Magdalena, Colombia. Trabajo de pregrado. Universidad del Magdalena, Santa Marta, Colombia.

Margules, C. R., \& Pressey, R. L. (2000). Systematic conservation planning. Nature, 405, 243-253.

Martín-Piera, F., \& Fernández-Torres, A. (1996). Coleópteros de la Sierra de Chiribiquete (Dpto. Caquetá, Colombia). Elytron Journal of the European Association of Coleopterology, 10, 23-50.

Martínez, B. G. (2009). Evaluación de la riqueza, abundancia, composición y biomasa relativa de coleópteros coprófagos (Scarabaeinae) en un paisaje agroforestal andino, Risaralda, Colombia. Trabajo de pregrado. Universidad de Caldas, Manizales, Colombia.

Martínez, N., García, H., Pulido, A., Ospino, D., \& Narváez, J. C. (2009). Fauna de escarabajos coprófagos (Coleoptera: Scarabaeinae) en un gradiente altitudinal en la vertiente Noroccidental de la Sierra Nevada de Santa Marta, Colombia. Neotropical Entomology, 38(6), 708-715.

Martínez, N., Cañas, L., Rangel, J., Barraza, J., Montes, J., \& Blanco, O. (2010a). Coleópteros coprófagos (Scarabaeidae: Scarabaeinae) en un fragmento de bosque seco tropical en el departamento del Atlántico, Colombia. Boletín del Museo de Entomología de la Universidad del Valle, 11(1), 21-30.

Martínez, N. J., Cañas, L. M., Rangel, J. L., Blanco, O., Mendoza, J. D., \& Cohen, S. (2010b). Coleópteros coprófagos (Scarabaeidae: Scarabaeinae) en la reserva natural Las Delicias (RND), Sierra Nevada de Santa Marta (SNSM), Colombia. Boletin Científico Centro de Museos Museo de Historia Natural, 14(2), 187-200.

Martínez, N., García, S., Gutiérrez, M., Sanjuan, S., \& Contreras, C. (2010c). Composición y estructura de la fauna de escarabajos (Insecta: Coleoptera) atraídos por trampas de luz en la reserva ecológica de Luriza, Atlántico, Colombia. Boletín de la Sociedad Entomológica Aragonesa, 47, 373-381.

Martínez-Quintero, B. G., Cultid-Medina, C. A., \& RudasGrajales, J. C. (2013). Metodo para marcar escarabajos coprófagos (Coleoptera: Scarabaeinae) y su implementación en los Andes de Colombia. Acta Zoologica Mexicana (n.s.), 29(2), 448-451.

Mcgeoch, M. A, Van Rensburg, B. T., \& Botes, A. (2002). The verification and application of Bioindicators: A case study of dung beetles in a Savanna ecosystem. The Journal of Applied Ecology, 39, 661-672. 
Medina, C. A., \& Kattan, G. H. (1996). Diversidad de coleópteros coprófagos (Scarabaeidae) de la reserva forestal de Escalerete. Cespedesia, 21(68), 89-102.

Medina, C. A., \& Lopera, A. (2000). Clave ilustrada para la identificación de los géneros de escarabajos coprófagos (Coleoptera: Scarabaeinae) de Colombia. Caldasia, 22(2), 299-315.

Medina, C. A., \& Pulido, L. A. (2009). Escarabajos coprófagos (Coleoptera: Scarabaeinae) de la Orinoquía colombiana. Biota Colombiana, 10(1, 2), 55-62.

Medina, C. A., Escobar, F., \& Rojas, A. M. (1990). Aspectos ecológicos y biomecánicos de Oxysternon conspicillatum (Col: Scarabaeidae) en el Valle del Cauca. Revista Colombiana de Entomología, 16(2), 54-61.

Medina, C. A., Lopera, A., Vítolo, A., \& Gill, B. (2001). Escarabajos coprófagos (Coleoptera: Scarabaeidae: Scarabaeinae) de Colombia. Biota Colombiana, 2(2), 131-144.

Medina, C. A., Escobar, F., \& Kattan, G. H. (2002). Diversity and habitat use of dung beetles in a restored Andean landscape. Biotropica, 34(1), 181-187.

Molina, L. J. (2005). Patrones de diversidad de la comunidad de escarabajos coprófagos (Coleoptera, Scarabaeidae, Scarabaeinae) en la zona cafetera, Quindío, Colombia. Trabajo de pregrado. Universidad Nacional de Colombia, Bogotá, Colombia.

Molina, L. J. (2007). Patrones espaciales de diversidad de la comunidad de escarabajos coprófagos en paisajes modificados. Armenia, Colombia: Ed. Elizcom.

Molano, F., \& Medina, C. A. (2010). Especie nueva de Scybalocanthon (Coleoptera: Scarabaeinae: Canthonini) y descripción de la variación del órgano genital masculino. Revista Mexicana de Biodiversidad, 81, 689-699.

Molano, F., \& Morales, I. (2006). Escarabajos coprófagos (Coleoptera: Scarabaeinae) asociados al excremento del mono aullador (Alouatta seniculus) de la Reserva Natural La Montaña del Ocaso, Quimbaya, Quindío. In Riqueza Biótica Quindíana, Armenia (pp. 395 398). Universidad del Quindío, Armenia, Colombia.

Montes, J. M. (2010). Efecto de borde en ensamblajes de escarabajos coprófagos (Coleoptera: Scarabaeidae) en fragmentos de bosque en el nordeste antioqueño, Colombia. Tesis de Maestría. Universidad Nacional de Colombia, Medellin, Colombia.

Moreno, C. J. (2009). Estudio de la comunidad de escarabajos coprófagos (Coleoptera: Scarabaeidae: Scarabaeinae) en un gradiente bosque montanoecotono-pastizal en la Serranía de las Quinchas, Otanche, Boyacá. Trabajo de pregrado. Universidad Jorge Tadeo Lozano, Bogotá, Colombia.

Moreno-Pérez, R. A. (2011). Etograma del escarabajo coprófago Deltochilum hypponum (Buquet, 1844) (Scarabaeidae: Scarabaeinae) en la reserva natural
"Rogitama", Arcabuco (Boyacá - Colombia). Trabajo de pregrado. Universidad Pedagógica y Tecnológica de Colombia, Tunja, Colombia.

Morrone, J. J. (1994). On the identification of areas of endemism. Systematic Biology, 43, 438-441.

Motta, L., \& Loáiza, Y. (2004). Capacidad bioindicadora de escarabajos coprófagos en fragmentos de bosque y pastoreo Caquetá, Colombia. Trabajo de pregrado. Universidad de la Amazonía, Leticia, Colombia.

Murcia, B. (2008). Distribución estructural de la fauna de coleópteros (Scarabaeidae: Scarabaeinae) en un gradiente de sucesión del piedemonte caqueteño, Florencia Caquetá. Tesis de Maestría. Universidad Nacional de Colombia, Bogotá, Colombia.

Murgueitio, E., \& Giraldo, C. (2009). Sistemas silvopastoriles y el control del parásitos. Carta de Fedegan, $115,60-63$.

Murillo, D. A., Quiros, K., \& Rodríguez, A. (2010). Estudio preliminar de la composición de escarabajos copronecrófilos (Scarabaeidae), en la estación ambiental Tutunendo Quibdó, Chocó, Colombia. Investigación, Biodiversidad y Desarrollo, 29(1), 102-109.

Murillo, L. R. (2009). Estructura de la comunidad de coleópteros coprófagos (Scarabaeinae: Aphodiinae) en sistemas silvopastoriles de estratos múltiples, Corpoica, Turipana. Trabajo de pregrado. Universidad de Córdoba, Montería, Colombia.

Navarro, L., \& Roman, K. (2009). Comparación de la comunidad de escarabajos coprófagos (Coleoptera: Scarabaeidae: Scarabaeinae) en una zona de uso ganadero y en un relicto de bosque seco tropical del departamento de Sucre. Trabajo de pregrado. Universidad de Sucre, Sincelejo, Colombia.

Navarro, L., Roman, K., Goméz, H., \& Pérez, A. (2009). Primer registro de Digitonthophagus gazella (Fabricius, 1787) para el departamento de Sucre, Colombia. Revista Colombiana de Ciencias, 1(1), 60-64.

Navarro, I. L., Roman, A. K., Gómez, F. H., \& Pérez, H. A. (2011). Listado de escarabajos coprófagos (Coleoptera: Scarabaeidae: Scarabaeinae) de la Serrania de Coraza, Sucre (Colombia). Revista Colombiana de Ciencia Animal, 3(2), 262-268.

Narváez, C. P. (2002). Escarabajos saprófagos (Coleoptera: Scarabaeidae) en la vereda El Rosa, zona de amortiguación del Parque Nacional Natural Munchique, El Tambo, Cauca. Trabajo de pregrado. Fundación Universitaria de Popayán, Popayán, Colombia.

Neita, J. C., \& Escobar, F. (2011). The potential value of agroforestry to dung beetle diversity in the wet tropical forests of the Pacific lowlands of Colombia. Agroforestry Systems, 83(3), 1-11.

Neita, J. C., Pardo, L. C., Quinto, D., \& Cuesta, N. G. (2003). Los escarabajos copronecrófilos (Coleoptera, Scarabaeidae) en la parcela permanente de 
investigación en Biodiversidad (PPIB) en Salero, Unión Panamericana, Chocó. In F. García, Y. Ramos, J. Palacios, J. Arroyo, A. Mena \& M. González (Eds.). Salero Diversidad biológica de un bosque pluvial tropical (bp-T) (pp. 79-90). Universidad Tecnológica del Chocó, Grupo de Investigación en Recursos Vegetales, Instituto de Investigaciones Ambientales del Pacífico y Comunidad de Salero, Unión panamericana, Chocó, Colombia.

Nichols, E., Larsen, T., Spector, S., Davis, A. L., Escobar, F., Favila, M., \& Vulinec, K. (2007). Global dung beetle response to tropical forest modification and fragmentation: A quantitative literature review and meta-analysis. Biological Conservation, 137(1), 1-19.

Nichols, E., Spector, S., Louzada, J., Larsen, T., Amézquita, S., Favila, M. E., \& The Scarabaeinae Research Network. (2008). Ecological functions and ecosystems services provided by Scarabaeinae dung beetles. Biological Conservation, 141, 1461-1474.

Noriega, J. A. (2001). Estudio de la actividad diaria de colonización del recurso alimenticio, en una comunidad de escarabajos coprófagos (Coleoptera: Scarabaeidae), a lo largo de un gradiente altitudinal en la Sierra Nevada de Santa Marta. Trabajo de pregrado. Universidad de Los Andes, Bogotá, Colombia.

Noriega, J. A. (2002a). Aportes a la biología del escarabajo sudamericano Sulcophanaeus leander (Waterhouse, 1891) (Coleoptera: Scarabaeidae). Acta Zoológica Mexicana (n.s.), 87, 67-82.

Noriega, J. A. (2002b). Fírst report of the presence of the genus Digitonthophagus (Coleoptera: Scarabaeidae) in Colombia. Caldasia, 24(1), 213-215.

Noriega, J. A. (2004). Preliminary checklist of the scarab community (Coleoptera: Scarabaeidae) at CIEM, Tinigua National Park, Meta-Colombia. Field Studies of Fauna and Flora La Macarena Colombia, 14, $37-44$.

Noriega, J. A. (2008). Análisis de las provincias biogeográficas y áreas de endemicidad de los escarabajos coprófagos (Coleoptera: Scarabaeidae) en Colombia. Tesis de Maestría. Pontificia Universidad Javeriana, Bogotá, Colombia.

Noriega, J. A. (2009). Análisis de la diversidad de escarabajos coprófagos (Coleoptera: Scarabaeidae) en el departamento de Cundinamarca. Revista de Tecnología, 8(1), 83-89.

Noriega, J. A. (2012). Dung beetles (Coleoptera: Scarabaeinae) attracted to Lagothrix lagotricha (Humboldt) and Alouatta seniculus (Linnaeus) (Primates: Atelidae) dung in a colombian amazon forest. Psyche, 437589, 1-6.

Noriega, J. A., \& Acosta, A. (2011). Population size and dispersal of Sulcophanaeus leander (Coleoptera:
Scarabaeidae) on riverine beaches in the Amazonian region. Journal of Tropical Ecology, 27, 111-114.

Noriega, J. A., \& Botero-Trujillo, R. (2008). First report of whip scorpions (Arachnida: Uropygi) feeding on dung beetles (Coleoptera: Scarabaeidae). Boletin Sociedad Entomológica Aragonesa, 42, 451-452.

Noriega, J. A., \& Calle, J. C. (2008). Consumption of Gustavia hexapetala (Aublet) Smith (Lecythidales: Lecythidaceae) by the dung beetle Eurysternus plebejus Harold (Coleoptera: Scarabaeidae). The Coleopterist Bulletin, 62(4), 455-460.

Noriega, J. A., \& Fagua, G. (2009). Monitoreo de escarabajos coprófagos (Coleoptera: Scarabaeidae) en la región neotropical. In A. Acosta, G. Fagua \& A. M. Zapata (Eds.). Técnicas de Campo en Ambientes Tropicales, manual para el monitoreo en ecosistemas acuáticos y artrópodos terrestres (pp. 165-188). Pontificia Universidad Javeriana, Bogotá, Colombia.

Noriega, J. A., \& Navarrete-Heredia, J. L. (2013). Quantification of predation on the dung beetle Canthidium cupreum (Blanchard) (Coleoptera: Scarabaeidae: Scarabaeinae) by Leistotrophus versicolor (Gravenhorst) (Coleoptera: Staphylinidae). The Coleopterist Bulletin, 67(2), 190-193.

Noriega, J. A., Solís, C., Quintero, I., Pérez, L. G., García, H. G., \& Ospino, D. A. (2006). Registro continental de Digitonthophagus gazella (Coleoptera: Scarabaeidae) en Colombia. Caldasia, 28(2), 379-381.

Noriega, J. A., Realpe, E., \& Fagua, G. (2007a). Diversidad de escarabajos coprófagos (Coleoptera: Scarabaeidae) en un bosque de galería con tres estadios de alteración. Universitas Scientiarum edición especial I, 12, 51-63.

Noriega, J. A., Solís, C., Escobar, F., \& Realpe, E. R. (2007b). Escarabajos coprófagos (Coleoptera: Scarabaeidae) de la Provincia de la Sierra Nevada de Santa Marta. Biota Colombiana, 8(1), 77-86.

Noriega, J. A., Botero, J. P., Viola, M., \& Fagua, G. (2007c). Dinámica estacional de la estructura trófica de un ensamblaje de Coleoptera en la Amazonía Colombiana. Revista Colombiana de Entomología, 33(2), 157-164.

Noriega, J. A., Cubillos, A. M., Castañeda, C., \& Sánchez, A. M. (2008a). Actividad diaria de colonización del recurso alimenticio en un ensamblaje de escarabajos coprófagos (Coleoptera: Scarabaeidae) en la Amazonía Colombiana. Acta Biológica Colombiana, 13(3), 75-86.

Noriega, J. A., Renjifo, J. M., \& Vaz-De-Mello, F. (2008b). First report of the genus Tetramereia Klages, 1907 (Coleoptera: Scarabaeidae: Phanaeini) in Colombia - notes to its distribution. Biota Colombiana, 9(1), 131-133. 
Noriega, J. A., Gillet, C. P. D. T., Sandoval-Mojica, A., Horgan, F. G., Blanco, J. I., Valencia, G., Pardo, L. C., \& Santibáñez, A. (2009). New records and distributional range extensión for Phanaeus haroldi Kirsch, 1871 (Coleoptera: Scarbaeidae) a consolidation of biological and biogeographic information. Lambillionea CIX, 109(4), 403-414.

Noriega, J. A., Moreno, J., \& Otavo, S. (2011). Quince años del arribo del escarabajo coprófago Digitonthophagus gazella (Fabricius, 1787) (Coleoptera: Scarabaeidae) a Colombia: proceso de invasión y posibles efectos de su establecimiento. Biota Colombiana, 12(2), 35-44.

Noriega, J. A., Palacio, J. M., Monroy-G., J. D., \& Valencia, E. (2012a). Estructura de un ensamblaje de escarabajos coprófagos (Coleoptera: Scarabaeinae) en tres sitios con diferente uso del suelo en Antioquia, Colombia. Actualidades Biologicas, 34(96), 43-54.

Noriega, J. A., Moreno, J., Otavo, S., \& Castaño, E. (2012b). New departmental records for Digitonthophagus gazella (Coleoptera: Scarabaeinae) in Colombia. Acta Biológica Colombiana, 17(1), 201-204.

Olarte, J. E. (2008). Coleópteros coprófagos (Coleoptera: Scarabaeidae) en parcelas de palma aceitera (Elaeis guineensis Jacq.) en el sur del Cesar. Trabajo de pregrado. Universidad Industrial de Santander, Bucaramanga, Colombia.

Orozco, J., \& Pérez, M. (2008). Escarabajos coprófagos (Coleoptera: Scarabaeoidea) del Parque Nacional Natural Los Estoraques (Norte de Santander, Colombia). Revista Brasileira de Entomologia, 52(1), 36-40.

Otavo, S. (2010). Escarabajos (Coleoptera: Scarabaeoidea) como posibles elementos indicadores del estado de conservación de los bosques amazónicos: Estudio de caso en el Parque Nacional Natural Amacayacu. Trabajo de pregrado. Universidad Distrital, Bogotá, Colombia.

Otavo, S., Parrado-Rosselli, A., \& Noriega, J. A. (2013). Superfamilia Scarabaeoidea (Insecta: Coleoptera) como elemento bioindicador de perturbación antropogénica en un parque nacional amazónico. Revista de Biologia Tropical, 61(2), 735-752.

Padilla-Gil, D. N., \& Halffter, G. (2007). Biogeography of the areas and Canthonini (Coleoptera: Scarabaeidae) of Dry Tropical Forests in MesoAmerica and Colombia. Acta Zoologica Mexicana (n.s.), 23(1), 73-108.

Palacio, J. (2007). Estudio de tres diferentes usos del suelo y su efecto en la estructura y funcionalidad del ensamblaje de escarabajos coprófagos (Coleoptera: Scarabaeidae) en el departamento de AntioquiaColombia. Trabajo de pregrado. Pontificia Universidad Javeriana, Bogotá, Colombia.

Pardo, L. C. (1997). Muestreo preliminar de los escarabajos copronecrófilos (Coleoptera: Scarabaeidae) de las selvas de la Fragua, cuenca baja del rio Calambre (Valle). Cespedesia, 22(69), 59-80.
Pardo, L. C., \& Castillo, L. D. (2002a). Contribución al estudio de los escarabajos copronecrofilos (Col. Scarabaeidae) de la quebrada río azul, Calima, Chocó biogeográfico, Colombia. Boletín Cientifico Museo Historia Natural Universidad de Caldas, 6, 161-176.

Pardo, L. C., \& Castillo, L. D. (2002b). Muestreo preliminar de los escarabajos copronecrófilos (Coleoptera-Scarabaeidae) de las selvas de chancos, Calima, Chocó biogeográfico (Valle). Boletín Científico Museo Historia Natural Universidad de Caldas, 6, 11-27.

Pardo, L. C., \& Rubiano M. (1994). Registros y observaciones preliminares de los escarabajos (Coleoptera-Scarabaeoidea) del páramo Las Hermosas, Valle-Tolima (Colombia). Cespedesia, 20(64-65), 87-114.

Pardo, L. C., Reyes, L. C., \& Franco, P. (1995). Escarabajos (Coleoptera: Scarabaeoidea) de la cuenca alta del río Pance, Farallones de Cali, Valle. I. Cespedesia, 20(66), 189-197.

Pardo, L. C., Arroyo, J. E., \& Quiñónez, F. (2004). Observaciones de los escarabajos copronecrófagos y sapromelífagos de San Luis Robles, Nariño. Boletín Cientifico Centro de Museos Museo de Historia Natural, 8, 113-139.

Pardo-Locarno, L. C. (2007). Escarabajos coprófagos (Coleoptera: Scarabaeidae) de Lloró, departamento del Chocó, Colombia. Boletín Cientifico Centro de Museos Museo de Historia Natural, 11, 377-388.

Pardo-Locarno, L. C., \& Henao, E. (1996). Noticia y prioridades investigativas de los escarabajos (ColeopteraScarabaoidea) del ecotono selvatico rio Dovio, Chocó Biogeográfico, Valle, Colombia. Cespedesia, 21(68), 133-146.

Pérez, G., \& Erazo, M. C. (2008). Escarabajos \& cucarrones. In J. V. Rodríguez-Mahecha, J. V. RuedaAlmoncid \& T. D. Gutiérrez (Eds.). Guía ilustrada de la fauna del Santuario de vida silvestre Los Besotes, Valledupar, Cesar, Colombia (pp. 117-168). Serie de guías tropicales de campo No. 7, Conservación Internacional-Colombia. Bogotá, Colombia: Editorial Panamericana, Formas e Impresos.

Pérez, M. (2003). Escarabajos coprófagos (Col: Scarabaeoidea, Scarabaeinae) del área Natural Única los Estoraques una aproximación a su estado y su uso como bioindicadores. Trabajo de pregrado. Universidad Francisco de Paula Santander, Ocaña, Colombia.

Pimm, S. L., Russell, G. J., Gittleman, J. L., \& Brooks, T. M. (1995). The future of biodiversity. Science, 269, 347-350.

Pineda, J. H., \& Vanegas, D. A. (2012). Caracterización de la comunidad de escarabajos coprófagos (Scarabaeidae, Scarabaeinae) en áreas naturales del Departamento del Quindío. Trabajo de pregrado. Universidad del Quindio, Armenia, Colombia. 
Posada, A. (2004). Escarabajos copro-necrófagos como indicadores de calidad biótica de fragmentos de bosque, mediante muestreos rápidos, municipio de El Retiro (Antioquia). Trabajo de pregrado. Universidad Nacional de Colombia, Medellin, Colombia.

Pulido-H., A. (2006). Escarabajos coprófagos (Coleoptera: Scarabaeidae: Scarabaeinae). In Caracterización de la biodiversidad proceso corredor biológico entre los PNN Puracé y Cueva de los Guácharos (Huila), Colombia (pp. 90-103). Villa de Leiva, Colombia: Grupo GEMA, Instituto de Investigación de Recursos Biológicos Alexander von Humboldt.

Pulido, L. A., Riveros, R. A., Gast, F., \& Von Hildebrand, P. (2003). Escarabajos coprófagos (Coleoptera: Scarabaeidae: Scarabaeinae) del Parque Nacional Natural "Serranía de Chiribiquete", Caquetá, Colombia (Parte I). m3m-Monografias Tercer Milenio (SEA), 3, 51-58.

Pulido, L. A., Medina, C. A., \& Riveros, R. A. (2007). Nuevos registros de escarabajos coprófagos (Scarabaeidae: Scrabaeinae) para la región Andina de Colombia. Parte I. Revista de la Academia Colombiana de Ciencias Exactas, Físicas y Naturales, 31(119), 305-310.

Quintero. S. I. (1998). Composición, diversidad y preferencias por recurso alimenticio en una comunidad de escarabajos coprófagos en una área de selva amazónica en Leticia - Amazonas - Colombia (Coleoptera: Scarabaeidae: Scarabaeinae). Trabajo de pregrado. Universidad Pedagógica Nacional, Bogotá, Colombia.

Quintero, I., Osorio, P., Castillo, R., \& Higuera, M. (2007). Insectos. In H. Villarreal-Leal \& J. MaldonadoOcampo (Eds.), Caracterización biológica del Parque Nacional Natural El Tuparro (Sector Noreste), Vichada Colombia (pp. 87-122). Instituto de Investigación de Recursos Biológicos Alexander von Humboldt. Bogotá, Colombia.

Redondo, A. R., \& Herazo, C. J. (2011). Estructura de un ensamblaje de escarabajos coprófagos (Coleoptera: Scarabaeidae), en tres hábitats diferentes, Sucre - Colombia. Trabajo de pregrado. Universidad de Sucre, Sincelejo, Colombia.

Regalado, A. (2013). Venturing back into Colombia. Science, 341(6145), 450-452.

Rivera, C. (2004). Biodiversidad de coleópteros copronecrófagos (Scarabaeidae: Scarabaeinae) en Bosque Seco Tropical, Sucre-Colombia. Trabajo de pregrado. Universidad de Antioquia, Medellín, Colombia.

Rivera, C., \& Wolff, M. (2007). Digitonthophagus gazella (Coleoptera: Scarabaeidae): Distribución en América y dos nuevos registros para Colombia. Revista Colombiana de Entomología, 33(2), 190-192.

Rodríguez, S. L. (2008). Evaluación de la diversidad de escarabajos coprófagos (Coleoptera: Scarabaeidae: Scarabaeinae) en seis formaciones vegetales en la selva de Matavén - Vichada. Trabajo de pregrado.
Universidad Pedagógica y Tecnológica de Colombia, Tunja, Colombia.

Rosado, L. I. (2008). Estudio de la comunidad de coleópteros coprófagos (Coleoptera: Scarabaidae-Scarabaeinae) en la Serranía de los Yariguíes (Santander - Colombia). Trabajo de pregrado. Universidad Industrial de Santander, Bucaramanga, Colombia.

Ruíz, F. (2008). Estudio del efecto de la presencia de un relleno sanitario sobre la estructura de un ensamblaje de escarabajos coprófagos (Coleoptera: Scarabaeidae). Trabajo de pregrado. Pontificia Universidad Javeriana, Bogotá, Colombia.

Salcedo, G., \& Sierra, K. (2010). Escarabajos coprófagos (Coleoptera: Scarabaeinae) asociados a excrementos de mono (Alouatta seniculus), cerdo (Sus domestica) y vaca (Bos taurus) en dos sectores del departamento del Atlántico, Colombia. Trabajo de pregrado. Universidad del Atlántico, Barranquilla, Colombia.

Sánchez, A. M., Aide, T. M., Clark, M. L., \& Etter, A. A. (2012). Land cover change in Colombia: Surprising forest recovery trends between 2001 and 2010. Plos One, 7(8), 43943.

Santos, M. C. (2007). Efectos de la dispersión secundaria por coleópteros coprófagos sobre el destino de semillas de Rollinia edulis (Annonaceae) dispersadas por dos especies de monos en la Serranía las Quinchas, Colombia. Trabajo de pregrado. Universidad Industrial de Santander, Bucaramanga, Colombia.

Santos-Heredia, C., Andresen, E., \& Zarate, D. A. (2010). Secondary seed dispersal by dung beetles in a Colombian rain forest: effects of dung type and defecation pattern on seed fate. Journal of Tropical Ecology, 26(4), 355-364.

Sarmiento-Garcés, R., \& Amat-Garcia, G. (2009). Escarabajos del género Dichotomius Hope 1838 (Scarabaeidae: Scarbaeinae) en la Amazonía colombiana. Revista de la Academia Colombiana de Ciencias, 33(127), 285-296.

Scarabnet. (2009). ScarabNet (Scarabaeinae Research Network) Global Taxon Database. Version 1.5, www. scarabnet.org.

Schoolmeesters, P. (2010). World Scarabaeidae database. In F.A. Bisby, M. A. Ruggiero, Y. R. Roskov, M. Cachuela-Palacio, S. W. Kimani, P.M. Kirk, A. Soulier-Perkins, \& J. van Hertum (Eds.), Species 2000 \& ITIS Catalogue of Life: 2010 Annual Checklist CDRom, Species 2000: Reading, U.K.

Semana. (2013). Los parques: de las balas a la paz. Revista Semana, 21 de Septiembre, http://www.semana.com//nacion/articulo/ los-parques-de-las-balas-la-paz/358371-3.

Solís, C. (2005). Composición y distribución de la comunidad de coleópteros coprófagos (Scarabaeidae: Scarabaeinae) en remanentes de bosque seco tropical 
(Bs-T) Departamento del Atlántico Colombia. Trabajo de pregrado. Universidad del Atlántico, Barranquilla, Colombia.

Solis, C., Noriega, J. A., \& Herrera, G. (2011). Escarabajos coprófagos (Coleoptera: Scarabaeinae) en tres bosques secos del departamento del Atlántico-Colombia. Boletín del Museo de Entomología de la Universidad del Valle, 12(1), 33-41.

Spector, S. (2006). Scarabaeine dung beetles (Coleoptera: Scarabaeidae: Scarabaeinae): an invertebrate focal taxón for biodiversity research and conservation. The Coleopterist Bulletin Monograph Number, 5, 71-83.

Torres, C. A. (2002). Riqueza y abundancia de los escarabajos copronecrófilos (Col: Scarabaeoidea, Scarabaeinae) en tres circunstancias ambientales en el bosque muy húmedo montano bajo, (bmhmb) de la vereda Cataluña Pereira, Risaralda, Colombia. Trabajo de pregrado. Programa de Agronomía, Corporación Universitaria Santa Rosa de Cabal, Pereira, Colombia.

Uribe, M. L. (2012). Escarabajos bioindicadores del bosque seco tropical del departamento de Caldas-Colombia. Trabajo de pregrado. Ingeniería Agronómica, Universidad de Caldas, Manizales, Colombia.

Vargas, L. V., \& Velandia, N. A. (2011). Reconocimiento de las especies de la subfamilia Scarabaeinae (Coleoptera: Scarabaeidae) de la Reserva Natural El Aguacate (Acandí- Chocó- Colombia) y diseño de una guía como estrategia didáctica para su conocimiento y valoración. Trabajo de pregrado. Universidad Pedagógica Nacional, Bogotá, Colombia.

Vargas-Pérez, A. (2013). Respuesta del ensamblaje de escarabajos coprofagos (Coleoptera: Scarabainae) a la transformación del hábitat en una zona de bosque seco tropical en el departamento de Córdoba, Norte de Colombia. Trabajo de pregrado. Universidad de Cordoba, Monteria, Colombia.

Vaz-De-Mello, F. Z. (2008). Synopsis of the new subtribe Scatimina (Coleoptera: Scarabaeidae: Scarabaeinae: Ateuchini), with descriptions of twelve new genera and review of Genieridium, new genus. Zootoxa, 1955, 1-75.

Villada-Bedoya, S. (2011). Evaluación de la diversidad de escarabajos coprófagos a través del ecotono bosque - café de sol en un paisaje cafetero colombiano, Risaralda. Trabajado de pregrado. Universidad de Caldas, Manizales, Colombia.

Villareal, H., Álvarez, M., Córdoba, S., Escobar, F., Fagua, G., Gast, F., Mendoza, H., Ospina, M., \& Umaña, A. M. (2004). Manual de métodos para el desarrollo de inventarios de biodiversidad. Instituto de investigación de recursos Biológicos Alexander von Humboldt, Bogotá, Colombia.

Vítolo, A. L. (2000a). Clave para la identificación de los géneros y especies phanaeinas (Coleoptera: Scarabaeidae: Coprinae: Phanaeini) de Colombia. Revista de la Academia Colombiana de Ciencia, 24(93), 591-601.

Vítolo, A. L. (2000b). Los escarabajos Phanaeini (Coleoptera: Scarabaeidae: Coprinae) de Colombia. Trabajo de pregrado. Pontificia Universidad Javeriana, Bogotá, Colombia.

Vítolo, A. L. (2004). Escarabajos estercoleros de la tribu Phanaeini de Colombia (Coleoptera: Scarabaeoidea: Scarabaeidae). In F. Fernández, M. G. Andrade, \& G. D. Amat (Eds.). Insectos de Colombia (Vol. 3, pp. 277-318). Universidad Nacional de Colombia, Bogotá, Colombia. 
